Research Square
Preprints are preliminary reports that have not undergone peer review.

They should not be considered conclusive, used to inform clinical practice, or referenced by the media as validated information.

\title{
Corpse Degradation of Farmed Fish Increases the Abundance of Antibiotic Resistance Genes but Exhibits the Temporal Stability
}

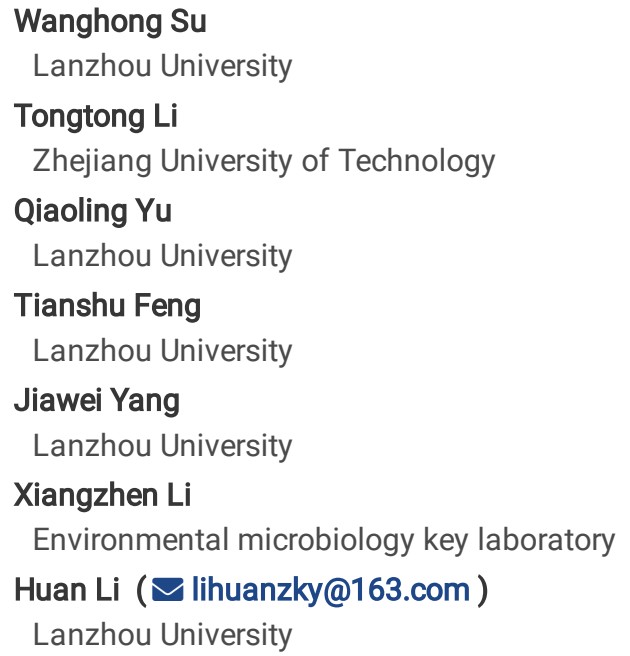

\section{Research Article}

Keywords: resistomes, temporal persistence, opportunistic pathogens, corpse decomposition, water pollution

Posted Date: August 11th, 2021

DOI: https://doi.org/10.21203/rs.3.rs-749577/v1

License: (c) (i) This work is licensed under a Creative Commons Attribution 4.0 International License. Read Full License 


\section{Abstract}

Serious concerns have been raised regarding resistomes causing by corpse decomposition in the aquatic environment, which pose threats to the water environment and human health. However, antibiotic resistance genes (ARGs) in large-volume tap water and their temporal stability during corpse decay are poorly explored. Here, high-throughput quantitative polymerase chain reaction (HT-qPCR) and amplicon sequencing were applied to profile ARGs and bacterial communities in experimental and control groups containing $50 \mathrm{~L}$ of tap water at 7th, 15th and 100th day during corpse decomposition. We found that most of the ARGs in experimental groups had higher abundance compared with the control groups independent of time. And the absolute abundance of some ARGs in the carcass groups was even enriched by 259 to $413,640-$ folds. The tetracycline and beta-lactamase resistance genes of the experimental groups were obviously enriched compared with control groups, and the ARG profiles were convergent during different decay stages, which indicated the long-term persistence of ARGs. Treatment, dissolved oxygen (DO) and pH were three important factors determining ARG profiles during corpse decomposition. Twelve opportunistic pathogens, especially Burkholderia, Legionella and Halomonas, remarkably increased as decomposition proceeded. Network analysis showed that opportunistic pathogens were significantly associated with ARGs. Our results emphasize that corpse decay increases the abundance and diversity of ARGs in large-volume drinking water independent of time while exhibits temporal persistence of ARGs, thereby uncovering the harmful effects of animal cadavers. It also provides valuable suggestions for the risk assessment and management of source water caused by corpse decay.

\section{Introduction}

Antibiotic resistance genes (ARGs) are called as inherent characteristic of microorganisms (Wright and Poinar, 2012). Recently, ARGs have been considered as an emerging pollutant to public health (Yang et al., 2018). In China, at least 100,800 tons of antibiotics are inappropriately used by some industries such as the livestock industry and agriculture annually (Qian et al., 2018). As a result, ARGs is disseminated from those sources like animal wastes, sewage and hospitals (Tang et al., 2015). Moreover, due to the persistence of ARGs in water, ARGs eventually enrich high abundances in water ecosystems such as fresh water, estuarine and marine systems (Baquero et al., 2008; Czekalski et al., 2014; Marti et al., 2014; Zhu et al., 2017). Notably, ARGs in tap water has been found in 25 cities around the world (Han et al., 2020). It is because these ARGs cannot be removed by treatment of drinking water so that high abundances of these pollutants exist in tap water. Finally, the ARGs are gradually enriched over time. As a result, this situation can make large quantities of antibiotics ineffective, leading to certain human diseases untreatable. For the other thing, ARGs can be transmitted by ARG-carrying opportunistic bacterial pathogens and nonpathogenic bacteria via horizontal gene transfer (HGT) (Sidhu et al., 2020). Therefore, ARGs may increase the risk of some waterborne disease outbreaks (Ben et al., 2020).

Despite numerous researches investigating ARGs in water environment, few studies focus on ARGs related to corpse decomposition especially in water systems. Corpse decay is the process which organic substances are broken down into simpler organic matter. It has been reported that carcass can bring some certain bacteria into water from skin, fecal and gut microbiome, thus ARGs related to these microbiome would be transmitted to water systems (Carter et al., 2007). As a natural phenomenon, the decay process of carcass decomposition starts when an animal dies. The corpse in the water begins with an initial sinking, then the temperature of internal body falls, and the functions of immune system begins to lose (Metcalf et al., 2016). When the heart function starts to cessation and the internal oxygen decreases, the body gets gradually wet and the respiratory system and digestive tract become suitable to anaerobic microorganisms' propagation (Emmons et al., 2017). Next, under the internal pressure from gas gathering, some fluids of the body flow over from orifices and into the outside of the body when corpse bloats. Meanwhile microbiome would expand and continue to break up outside the body. Later, the decomposition of the body debris gradually slows down over time (Carter et al., 2007). Obviously, the decomposition course can be divided into five different phases, which includes early floating, submerged fresh, early floating, floating decay and sunken remains (Emmons et al., 2017; Zimmerman and Wallace, 2008). The submerged fresh, the floating decay and the sunken remains could represent before-decomposing, decomposing and after-decomposing conditions, thus this study focused on these three stages.

Corpse can produce many toxic materials such as nitrite, putrescine and cadaverine, which leave bad influence on both human health and water safety (Lobb et al., 2020; Parmenter and MacMahon, 2009). It is reported that almost $80 \%$ of corpse could be consumed by most microbes and insects (Spicka et al., 2011). Because of the input of nutrient resource mostly including carbon and nitrogen, the corpse degradation significantly influences environmental bacterial communities (Weiss et al., 2016). Generally, corpse decay is a complex phase associated with not only biological factors which include microbiotas and insects but non-biological factors like pH and conductivity (Guo et al., 2016). However, the biotic factors have a more important effect on carcass degradation (Metcalf et al., 2016).

Notably, microbial communities exhibit successional patterns during corpse decomposition (Lobb et al., 2020; Singh et al., 2018). The cadaver shows a dominant change from aerobic bacteria to anaerobic bacteria throughout decomposition process (Hyde et al., 2013). As a result, certain microbes can be used as indicators to more accurately determine death time point under specific environmental conditions (Heimesaat et al., 2012). Additionally, carcass decomposition may raise the potential risk of disease and pose a threat to human health. It is reported that

Page 2/21 
some opportunistic pathogens were detected in recreational water (Falkinham et al., 2015). For example, Pseudomonas could cause many diseases like septicemia, pneumonia and urinary tract infection, which are almost nosocomial infections (Young et al., 1982). As previous studies shown, some pathogens are detected in human remains (Conly and Johnston, 2005). Therefore, there is a potential risk between pathogens and human during corpse decomposition. Some water pathogens such as Coliforms and Salmonella in drinking water systems are detected in previous studies (Chen et al., 2019), and these bacteria usually relate to corpse decomposition. It is reported that a diarrheal outbreak affected over 1500 people happened which possibly resulted from the drinking water sources contaminated with Coliforms (Rai et al., 2019). So if the drinking water systems are polluted by these pathogens, there will be a threat for public suffer from gastroenteristis. At the same time, we suppose that ARGs could be carried by these pathogens and transferred to the drinking water systems, thereby causing greater risks of drug resistance and water-borne diseases. However, which potential water pathogens are associated with ARGs during corpse degradation remains unknown.

Recently, more and more studies have focused on the influencing factors of antibiotic resistance genes during corpse decomposition. Our previous study only paid attention to the ARGs' influencing factors in small-volume water system and short time scale (Zhou et al., 2021). However, there are still few studies regarding the ARGs during corpse degradation in large-volume water systems and long time scale. In fact, the large-volume drinking water system is closer to the reality of nature, and it is necessary to have a longer time to observe the impact factors and resilience of the ARGs at a longer time. So our study starts to focus on the resistomes in large-volume drinking water system during the whole decomposition (i.e. 7th -100th day) under dilution effect.

By high-throughput sequencing and high-throughput quantitative PCR, our research sought to explore temporal dynamic of ARG profiles, microbial communities and physicochemical factors during three important stages of corpse decomposition, which includes the stage early floating (at the 7th day of carcass decay), advanced floating decay (15th day) and sunken remains (100th day). In addition, fish (Carassius auratus) is chosen as a model as a result of aquaculture diseases' outbreak (Zhu et al., 2017). Carassius auratus, a common bulk freshwater aquaculture variety, are even produced nearly 2.0 million tons in china annually (Li et al., 2012). If these water-borne diseases due to the fish decompose outbreak, there will be a huge threat to human health and water environment. We chose tap water in a large volume as decomposition matrix to find the common changes of diverse types of water environments like river and pond. On the other hand, we mostly focus on the temporal variation of abundance and diversity of ARGs to give some advice for environmental pollution and management causing by ARGs and natural resilience of microbial community. Therefore, it is hypothesized that corpse decomposition changes ARG profiles and microbial communities. Here, four questions arise: (1) whether carrion degradation changes ARG profiles and bacterial communities over the decay process? (2) whether ARG profiles remain divergent or relatively stable over time? (3) which factors affect ARG profiles? (4) Which potential water bacterial pathogens are associated with ARGs?

\section{Materials And Methods}

\subsection{Experimental designing and water sampling}

This research was done from March to June 2016 in Chengdu, Sichuan Province, China ( $\left.30.67^{\circ} \mathrm{N}, 104.06^{\circ} \mathrm{E}\right)$. Our experiment was carried out for 100 days with three carcass decay stages which included the sunken remains (at the 100th day), advanced floating decay (15th day) and early floating (7th day). By Air conditioning, room temperature was controlled at around $25{ }^{\circ} \mathrm{C}$, while humidness was maintained at $40 \%$ during the 100 days of the experiment through air moistener. Carassius auratus was used as an animal decay model to study ARG profiles, bacterial community and water environmental factors with tap water. First, we prepared 30 sterile plastic boxes ( $\pi \times 0.2 \mathrm{~m}{ }^{2} \times 0.5 \mathrm{~m}, 50 \mathrm{~L}$ of tap water in each bucket) and divided randomly into six groups, which had five buckets in each group. We randomly selected three groups as experimental groups (KE, tap water with one fish carcass in each bucket, fish weight $80 \mathrm{~g} \sim 120 \mathrm{~g}$ ), and the remaining groups as control groups (KC, drinking water without carcass). Before the fish corpses were randomly placed into each of 15 boxes of the experimental groups, the euthanization of these fish was carried out by excess anesthetics Ethyl 3-aminobenzoate methanesulfonate (MS222, LOT: X21M9Y56602, Hunan Tiancheng polymer material co., Changsha, China) by over $600 \mathrm{mg} / \mathrm{L}$ dose within $10 \mathrm{~min}$.

Next, we collected water samples at three time points (7th day, 15th day and 100th day) and filtered these samples through filter device and pump machine (RICKY LABORATORY, China) with $0.45 \mu \mathrm{m}$ cellulose membranes used for experimental groups ( $250 \mathrm{~mL}$ water) and $0.22 \mu \mathrm{m}$ for control groups (250 mL water). The reason why we selected two membranes was for getting the sample faster. Then we used sealed plastic tubes to hold these filter membranes and stored in $-20{ }^{\circ} \mathrm{C}$. In order to analyze physicochemical property, we also collected $100-150 \mathrm{~mL}$ remaining water samples from each box into sterile tubes. Moreover, owing to these destructive sampling, the remaining plastic boxes and water should not be reused.

The animal treatment and experimental procedures were followed and permitted by the Animal Welfare and Ethics Committee of Chengdu Institute of Biology, Chinese Academy of Sciences (CIB-201602-174).

Page $3 / 21$ 


\subsection{Analysis of environmental factors}

The physicochemical parameters of water total nitrogen (TN), total organic carbon (TOC), ammonia nitrogen $\left(\mathrm{NH}_{4}-\mathrm{N}\right)$, nitrate-nitrogen $\left(\mathrm{NO}_{3}-\mathrm{N}\right)$, dissolved oxygen (DO), conductivity (CON), and $\mathrm{pH}$ were analyzed. Water $\mathrm{pH}$ and $\mathrm{CON}$ were identified by a bench $\mathrm{pH}$ water quality analyzer (AZ86505, shenzhen lemaiyi electronics co., LTD.), while DO value acquired by a dissolved oxygen meter (YSI59, YSI Corporation in China, USA). In addition, the $\mathrm{NH}_{4}-\mathrm{N}$ concentration was determined through Nessler's reagent colorimetric method (Ming-Xing et al., 2005) while $\mathrm{NO}_{3}-\mathrm{N}$ content by colorimetry (Wang et al., 2011). At last, using nondispersive infrared absorption method, water TOC was detected (Visco et al., 2005), and TN content through potassium persulfate UV spectrophotometry (Fan, 2011).

\subsection{DNA extraction and high-throughput sequencing}

Firstly, we extracted DNA from filter membrane (Beijing Solarbio Science \& Technology Co., Ltd, China) by Soil Ezup genomic DNA extraction kit (Sangon Biotech, Shanghai, China), then minimized PCR inhibitors through Geneclean Spin Kit (QBiogene, Carlsbad, CA). In addition, A260/A280 was measured by the Nanodrop 2000 Spectrophotometer (Thermo Scientific, IL, Waltham, USA). The genomic DNA extracts were served to subsequent PCR amplification of the V4-V5 regions of $16 \mathrm{~S}$ rRNA gene using the primer set $515 \mathrm{~F}$ (5'-GTGCCAGCMGCCGCGG-3') and 909R (5'-CCCCGYCAATTCMTT-TRAGT-3') (Li et al., 2016). The PCR mixture included $1 \mu \mathrm{L}$ Primer F/R, $2 \mu \mathrm{L}$ template DNA, $0.8 \mu \mathrm{L}$ of dNTPsmix, $1 \mu \mathrm{L}$ of $10 \times$ Toptaq Buffer and was added with double distilled water $\left(\mathrm{ddH}_{2} \mathrm{O}\right)$ to $25 \mu \mathrm{L}$. Notably, PCR was performed in 2 replicates per sample. The PCR processing was denatured at $94{ }^{\circ} \mathrm{C}$ for $3 \mathrm{~min}, 30$ cycles of $40 \mathrm{~s}$ at $94{ }^{\circ} \mathrm{C}$, and $1 \mathrm{~min}$ for annealing at $56{ }^{\circ} \mathrm{C}$, followed by an extension of $1 \mathrm{~min}$ at $72{ }^{\circ} \mathrm{C}$, and $72{ }^{\circ} \mathrm{C}$ for $10 \mathrm{~min}$. Using $1 \%$ agarose gel, the PCR products were run through electrophoresis and were implemented by SanPrep DNA Gel Extraction Kit (Sangon Biotech, China) for gel extraction (Li et al., 2016). After pooling all PCR products with equal molar, we used reagent Kit V2 of 250 bp paired-end Illumina MiSeq sequencer (Illumina, San Diego, CA, USA) for sequencing.

\subsection{Bioinformatics analysis}

We analyzed original sequences by Quantitative Insights Into Microbial Ecology (QIIME v1.9.0) (http://qiime.org/tutorials/tutorial.html) pipeline (Caporaso et al., 2010; Li et al., 2016). We merged these fastq paired-end original sequences through Flash.1.2.8 software (Magoč and Salzberg, 2011). Based on unique barcodes, we distinguished the sequences. And then, we excluded low-quality sequences (average value of base mass under 30 or $\mathrm{N}$-terminal sequence or length less than $300 \mathrm{bp}$ ) for the downstream analysis. Chimeras sequences were eliminated by denovo mode in Usearch 8.0 (Puente-Sánchez et al., 2015). According to UCLUST algorithm, nucleic similarity was $97 \%$ by operational taxonomic units (OTUs) cluster, and the longest sequences were selected as representative sequences. To acquired OTU table, the selected representative sequences were sorted up by Ribosomal Database Project (RDP) Classifier. These OTUs which did not belong to bacteria were excluded. In order to decrease the influence resulting from sequencing inequality, each sample was applied the "Daisychopper" script for resampling the OTU table (http://www.festinalente.me/bioinf/), generating 19,221 sequences. Finally, we used time-dependent trend curves through observed species (or OTUs) and phylogenetic diversity in order to evaluate community diversity. In addition, in order to ensure enough sequence, we obtained rarefaction curves from OTU level.

\subsection{High-throughput quantitative PCR analysis}

We used the WaferGen SmartChip Real-Time PCR System (Anhui, China) for target a total of 108 genes by HT-qPCR, including 16S rRNA, six transposases (tnpA-07, tnpA-05, tnpA-04, tnpA-02, tnpA-01, and IS613), 2 integrase genes, which covered intl-1(clinic), cIntl-1(class1), and 99 targeted ARGs which were thought to confer resistance to major class of resistance (Su et al., 2018)such as aadA-01 and floR. Table.S1 showed the primer sets in this study (Wang et al., 2014). These PCR mixtures were first added to the microchip using 96 (samples) $\times 54$ (assays) mode of the SmartChip Multisample Nanodispenser (MSND), followed by qPCR reaction on Cycler. A total of 2 chips were run, 30 samples and 54 pairs of primers were run on a single chip, among which 30 samples and 1 NTC were repeated for triplicate. We analyzed sample with a $100 \mathrm{~nL}$ reaction volume, including $50 \mathrm{~nL}$ 1×LightCycle 480 SYBR Green IMaster (Roche Applied Sciences), $200 \mathrm{ng}$ DNA template, $500 \mathrm{nM}$ each primer and $19 \mathrm{~nL}$ dd $_{2} \mathrm{O}$. The conditions were as follows: denaturation $10 \mathrm{~min}$ at $95^{\circ} \mathrm{C}, 40$ amplification cycles $30 \mathrm{~s}$ at $95^{\circ} \mathrm{C}$ and extension $30 \mathrm{~s}$ at $60^{\circ} \mathrm{C}$. Because of occurrence of negative results, we selected available standard curves which $R^{2}$ value was 0.990 - 0.998 and amplification efficiency was $90 \%-110 \%$ (Su et al., 2018). We analyzed results automatically on the SmartChip qPCR software (version 2.7.0.1). Additionally, the detection limit was set as 31 of threshold cycle value $\left(\mathrm{C}_{\mathrm{T}}\right)$. We calculated gene copy number by comparing $\mathrm{C}_{\mathrm{T}}$ value based on equation (Ouyang et al., 2015). In addition, we calculated the relative abundance by dividing 16S rRNA gene copies (Cui et al., 2018). Meanwhile, according to gene copy numbers and 16S rRNA absolute abundance, absolute abundance (gene copy numbers per liter of water) was acquired (Liu et al., 2018).

\subsection{Statistical analysis}

All charts were done using Origin 2018 (Originlab, Northampton, USA). And we used One-way analysis of variance (One-way ANOVA) with Tukey's posthoc test at SPSS21.0 for observing the variation of environmental factors, relative abundances of opportunistic pathogens and alpha diversity between experimental and control groups. Alpha diversity was estimated by four diversity metrics: Chao1 and Observed OTUs.

Page 4/21 
The temporal distribution of ARGs, mobile genetic elements (MGEs), and bacterial taxa at genus and OTUs levels in the experimental group was presented through ternary phase diagram by Origin 2018. The percentage stacking bar chart was created using Origin 2018 to describe bacterial communities. The QIIME script "otu_category_significance.py" was applied to identify the difference of bacterial taxa between control and experimental groups. And according to R 3.6.2 (R Foundation for Statistical Computing, Vienna, Austria) with pheatmap package, the difference among ARGs, MGEs, microbial abundance and 12 conditioned pathogens in different groups was represented by heatmap. We described communities' structure with unweighted and weighted UniFrac distance matrices by using QIIME and Origin 2018. Unweighted UniFrac distance depended on the basis of phylogenetic relationship and OTUs' species abundance, while the species absence/presence and phylogenetic relationship were considered by weighted Unifrac (Lozupone and Knight, 2005). In addition, we applied Permutational multivariate analysis of variance (PERMANOVA) (Balakrishnan et al., 2014) to observe the effects of treatment and time factors in ARGs, and shaping microbial community members and structures. And we described ARG profiles by principal coordinates analysis (PCoA) using QIIME1.9.0 (Zhou et al., 2021). And we also describe unique and common genes of ARGs across all samples by Venn Diagram (https://bioinfogp.cnb.csic.es/tools/venny/index.html). In addition, we calculated the deterministic change caused by the treatment can be approximated by $\mathrm{D}=$ [(mean compositional variation between treatment and control) - (mean compositional variation between control and control)] at three time points. Similarly, the stochastic change caused by treatment can be approximated by $S=[($ mean compositional variation within treatment) - (mean compositional variation within control] at each three time points. Therefore, the relative importance of the deterministic change $=|\mathrm{D}| /(|\mathrm{D}|+|\mathrm{S}|)$. The more detailed calculation methods to describe stochastic, deterministic change of ARG compositions were described in the reference (Zhang et al., 2016).

To analyze the correlation among target ARGs, dominant bacterial taxa at genus and OTUs levels (mean relative abundance $>1 \%$ ) and physicochemical factors, we used pearson analysis of SPSS21.0 and R3.6.2 to acquire heatmap. We used Origin 2018 to generated linear fitting between all ARGs and MGEs. Furthermore, we analyzed the effects of treatment, time, microbe, physicochemical factors and MGEs on ARGs by PERMANOVA and multiple regression on matrices (MRM) (Deng et al., 2016). Finally, we further analyzed these results to generate the network analysis $(P<0.05,|r|>0.6)$ using R 3.6.2 and Gephi software v. 0.9.2 (Gephi, WebAtlas, France) (Cherven, 2013).

\section{Results}

\subsection{Variation of water environmental factors during carcass decay}

All water environmental properties including $\mathrm{TN}, \mathrm{TOC}, \mathrm{NO}_{3}-\mathrm{N}, \mathrm{NH}_{4}-\mathrm{N}, \mathrm{DO}, \mathrm{CON}$ and $\mathrm{pH}$ were significantly affected by treatment (or corpse degradation) (Fig.S1区 Table S2, One-way ANOVA, $P<0.01$ ). Compared with control groups, $\mathrm{pH}, \mathrm{DO}$ and $\mathrm{NO}_{3}-\mathrm{N}$ in experimental groups decreased significantly (Fig.S1-a, c, e, One-way ANOVA, $P<0.01$ ). However, $\mathrm{CON}, \mathrm{NH}_{4}-\mathrm{N}, \mathrm{TOC}$ and TN in experimental groups increased significantly (Fig.S1-b, d, f, g, One-way ANOVA, $P<0.001$ ). In addition, $\mathrm{NH}_{4}-\mathrm{N}, \mathrm{CON}$ and $\mathrm{pH}$ in the experimental groups showed a growing trend during corpse decomposition (Fig.S1-a, b, d). On the contrary, DO, TOC, TN and $\mathrm{NO}_{3}-\mathrm{N}$ in experimental groups showed a downward trend (Fig.S1-c, e, f, g). And only $\mathrm{NH}_{4}-\mathrm{N}, \mathrm{CON}$ and $\mathrm{pH}$ were significantly affected by time (Table S2, Two-way ANOVA, $P<0.001$ ). The interaction between corpse degradation and time also had significant effects on the concentrations of CON, TOC and NH${ }_{4}-\mathrm{N}(\mathrm{Table} \mathrm{S2}$, One-way ANOVA, $P<0.01)$.

\subsection{Diversity and abundance of ARGs during corpse decomposition}

Among all 108 target genes, only 8 MGEs (including two integrase and six transposases) and 56 different ARGs were detected in all water samples. ARGs were classified into 9 types which included vancomycin, sulfonamide, beta-lactamase, aminoglycoside, chloramphenicol, Macrolide-Lincosamide-Streptogramin B resistance genes (MLSB), multidrug, tetracycline, and others (Fig.S3-a). And compared with control groups, the number of tetracycline, beta-lactamase, and vancomycin genes in carcass groups increased (Fig.S3-a). ARGs in all samples were divided into four resistance mechanisms which included cellular protection (43.87\%), antibiotic deactivate (36.04\%), efflux pump (16.25\%), and others (3.84\%) (Fig.S3-b).

Notably, the absolute abundance of all MGEs and ARGs was significantly impacted by corpse degradation (Table 1, Two-way ANOVA, $P \leq$ 0.001). Time or the interaction between treatment and time also posed an impact on absolute abundance of most resistomes, which included vancomycin, tetracycline, sulfonamide, multidrug, MLSB, chloramphenicol, and beta-lactamase (Table 1, $P<0.05$, Two-way ANOVA). However, these two factors insignificantly affected on MGEs' absolute abundance $(P>0.05$, Two-way ANOVA). Multidrug resistance genes' absolute abundance was mostly affected by treatment (i.e. corpse decomposition) $(\mathrm{F}=154.190, P<0.001)$, time $(\mathrm{F}=21.787, P<0.001)$ and their interaction $(F=21.785, P<0.001)$. Similarly, treatment had a significant effect on the relative abundance of aminoglycoside, vancomycin, tetracycline, and sulfonamide (all $P<0.05$, Two-way ANOVA). Time posed a significant impact on the relative abundance of aminoglycoside, beta-lactamase, multidrug, sulfonamide, tetracycline and vancomycin $(P<0.05$, Two-way ANOVA). The interaction between treatment and time had a significant effect on the relative abundance of beta-lactamase, chloramphenicol, MLSB, tetracycline and vancomycin $(P<0.05$, 
Two-way ANOVA). Compared with absolute abundance (PERMANOVA, $R^{2}=0.363, P=0.001$ ), cadaver degradation had a greater impact on the relative abundance (Table 2, PERMANOVA, $R^{2}=0.428, P=0.001$ ). Although time had no significant effect on the whole ARG profiles in all samples (Table 2, $P>0.05$ ), time posed significant effect on the relative abundance of ARG profiles in experimental groups (PERMANOVA, $R^{2}=$ $0.363, P=0.029$ ). In addition, time had significant effect on the absolute abundance of 24 ARGs genes (i.e. acrA-05, acrF and so on) and the relative abundance of 19 ARGs genes (such as aadA-01 and ampC-09) in experimental groups (Table S8, Table S9), while insignificant influenced most of ARGs' abundance (37 ARGs' relative abundance and 32 ARGs' absolute abundance). In other words, the abundance of most ARGs in experimental samples were not affected by time and maintained relatively stable in different corpse decay stages.

Thirty-seven shared ARG subtypes among three different copse subgroups persistently existed throughout decomposition process, and the proportion of these ARGs accounting for $68.5 \%$ proportion of ARGs in all corpse groups (Fig.S2). Interestingly, the number of the shared ARGs subtypes between control groups and experimental groups was only 14, but later was 29 and 26 at 7th, 15th and 100th day, respectively (Fig.S2). In addition, all the ARG profiles were significantly influenced by treatment at each time point (Fig.1-a, b, Table S3, PERMANOVA, $P<$ 0.05). And the effect of treatment increased firstly and decreased during decomposition, which reached the highest at 15th day (Table S3, PERMANOVA, absolute abundance $R^{2}=0.607$, relative abundance $R^{2}=0.759, P<0.05$ ). In addition, no significant difference was found among all control groups at different time points (Table S3, PERMANOVA, $P>0.05$ ), while significant difference was existed on 7KE vs. 15KE (Table S3, PERMANOVA, absolute abundance $R^{2}=0.255$, relative abundance $R^{2}=0.403, P<0.05$ ). Notably, the ARG profiles of the three experimental groups became more similar or converged over degradation time (Fig.1-a, b).

Both the absolute and relative abundance of the total ARGs in experimental increased significantly compared with control groups (Fig.2-a, b). In detail, the abundance of total ARGs increased firstly, and then decreased over time, but did not recover to level of control group yet. Additionally, the relative abundance of ARGs was enriched about 2-266 folds in experimental groups compared to the control group (Fig.2-d) while the enrichment multiple of these ARGs' absolute abundance was even 259-413,640 folds (Fig.2-c). In the experimental groups, some ARGs' absolute abundance such as tetB-02, aadA-02 and tetA-02 were enriched over 258, 59,194 and 413,639 times than the control groups, respectively. Obviously, most of ARGs in experimental groups displayed higher concentrations compared to the control groups, and remain a relatively stable abundance over decomposition (Fig.S4-a, b). After screening for significantly enriched ARGs of experimental groups, the abundance of ARGs genes such as tetB-01 and tetA-02 were significantly enriched in the cadaver groups in the three time points (i.e. 7 th day, 15 th day and 100th day) (Fig.3, Fig.S5). In addition, the abundance of MGEs such as intl-1(clinic) and tnpA-01 significantly enriched in experimental groups and slightly increased over decomposition stages (Fig.3, Fig.S6).

It was also shown the different distribution of ARGs and MGEs in corpse groups at 7th, 15th and 100th day of corpse decomposition (Fig.4-a, b, Fig.S7). The abundance of vancomycin genes was enriched at 7th day, 100th day while the abundance of tetracycline genes was enriched at 15th day (Fig.4-a, b). Besides, efflux pump was main resistance mechanism at 7th day and 15th day while cellular protection was main resistance mechanism at 100th day (Fig.S7).

In order to investigate the assembly mechanisms of ARG communities of drinking water, we govern stochastic and deterministic processes' relative importance during corpse decomposition (Fig.S3-c). Deterministic processes in corpse groups dominantly affected shape of ARG profiles at all the time points instead of stochastic processes (all compositional determinacy $>50 \%$ ), indicating treatment (i.e. corpse decay) may more influence the shape of ARG profiles than stochastic factors.

\subsection{Bacterial community composition and opportunistic pathogens during corpse decomposition}

We acquired 1,864,462 high-quality sequences across 30 samples (mean 62,149 reads each sample, $\mathrm{SD}=19,819$, $\min =14,843$, and $\max =$ $95,345)$, which were clustered into 11,053 unique OTUs with a threshold of $97 \%$ sequence similarity.

During the corpse decomposition, Proteobacteria and Bacteroidetes were main phyla (Fig.S8-a). Compared with control groups, the relative abundance of Bacteroidetes and Firmicutes increased significantly in corpse groups at the three time points (Fig.5-a, Fig S9-a, b, c, Table S4, $P$ $<0.001$, Two-way ANOVA). The Proteobacteria relative abundance in experimental groups gradually increased over time (Two-way ANOVA, $P<$ 0.001). At genus level, Blvii28 and Novispirillum dominated in experimental groups (Fig.S8-b). Blvii28 and Novispirillum relative abundance increased significantly compared with control groups (Fig.5-b, Fig S9-a, b, c, Table S5, $P<0.001$, Two-way ANOVA). In addition, Novispirillum abundance declined over time $(P<0.001$, Two-way ANOVA). In addition, the dominant phylum exhibited different distributions at three time points (Fig.4-C). The dominant genera in experimental groups were Sphingomonadales (UG), Nevskia and Azospira at 7th day, 15th day and 100th day, respectively. At OTU level, OTU14020_Blvii28 (UG) and OTU20104_Novispirillum (UG) in corpse groups increased significantly compared with control groups (Fig.5-c, Fig.S9-d, e, f). And OTU14020_Blvii28 (UG) slightly increased firstly but then decreased over time. The dominant OTU in experimental groups at 7th day, 15th day and 100th day were OTU31089_Acetobacteraceae (UG), OTU3597_Azospira (UG) and OTU48291_mitochondria respectively (Fig.4-d). 
Importantly, corpse decomposition had a significant effect on observed OTUs and chao1 index in three time points $(P<0.05$, One-way ANOVA) with the exception for observed OTUs at the 15th day ( $P>0.05$, One-way ANOVA, Fig.S8-c, d). Both Chao 1 index and observed OTUs in corpse groups gradually decreased over time (Fig.S8-c, d). Additionally, based on the unweighted and weighted UniFrac distance, there were significant shifts of community structures and members among all groups over time (Fig.1-c, d). Corpse decay significantly influenced the bacterial communities' structures and this effect became smaller over time (Table S5, PERMANOVA, weighted UniFrac at 7th day $R^{2}=0.935,15$ th day $R^{2}=0.896,100$ th day $\left.R^{2}=0.682, P<0.05\right)$. Notably, bacterial communities' dissimilarity of experimental groups became gradually smaller over time.

In addition, we screened 20 opportunistic pathogenic genera according to previous references (Table S6), 12 genera i.e. Comamonas, Bacteroides, Pseudomonas, Brevundimonas, Delftia, Mycobacterium, Legionella, Halomonas, Clostridium, Burkholderia, Aeromonas and Acinetobacter were detected in our dataset. The relative abundance of Bacteroides and Pseudomonas in experimental groups increased significantly compared with control groups (Fig.5-d). In addition, Bacteroides and Aeromonas in experimental groups gradually decreased over time, and Aeromonas gradually decreased to the level of the control groups at 100th day (Fig.S8-e, f, $P<0.05$ ).

\subsection{The influencing factors of ARG profiles during corpse decomposition}

For the relative and absolute abundance, ARG profiles were significantly impacted by treatment (i.e. corpse decomposition) (absolute abundance $R^{2}=0.363$, relative abundance $R^{2}=0.428$ ) and beta-diversity (absolute abundance $R^{2}=0.239$, relative abundance $R^{2}=0.287$, all $P$ $=0.001)$ (Table 2). The relative abundance of ARG profiles (i.e. observed OTUs) was only significantly shaped by alpha diversity $\left(R^{2}=0.083, P\right.$ < 0.01). In addition, seven environmental factors (i.e. $\mathrm{TN}, \mathrm{TOC}, \mathrm{NO}_{3}-\mathrm{N}, \mathrm{NH}_{4}-\mathrm{N}, \mathrm{DO}, \mathrm{CON}$, and $\mathrm{pH}$ ) were also significant impacting factors shaping the ARG profiles $(P<0.01)$. In addition, we found ARGs' absolute abundance were significantly related to some MGEs (i.e. tnpA-07, tnpA-05, tnpA-04, tnpA-02, tnpA-01, and intl-1(class1)) $(P<0.01)$. Meanwhile, ARGs' relative abundance were significantly correlated to certain MGEs (i.e. tnpA-05, tnpA-01, intl-1(class1), and clntl-1(class1)) $(P<0.05)$. Among these factors, we found the most affecting factors related to the absolute abundance were treatment $\left(R^{2}=0.363\right)$, DO $\left(R^{2}=0.359\right)$ and intl-1(class 1$)\left(R^{2}=0.267\right)$, while the most influencing factors associated with the relative abundance were treatment $\left(R^{2}=0.428\right), \mathrm{DO}\left(R^{2}=0.425\right)$ and $\mathrm{pH}\left(R^{2}=0.317\right)$.

And then, we divided these influencing factors into 5 parts (i.e. time, MGEs, environmental factors, microbial community and treatment) (Table S7). For the absolute abundance, treatment $\left(R^{2}=0.648\right)$, MGEs $\left(R^{2}=0.190\right)$ and environmental factors $\left(R^{2}=0.158\right)$ were significantly associated with ARGs (MRM, $P<0.01)$. And for the relative abundance, treatment $\left(R^{2}=0.544\right)$, environmental factors $\left(R^{2}=0.356\right)$ and microbial community $\left(R^{2}=0.015\right)$ were significantly linked with ARGs (MRM, $\left.P<0.01\right)$. Notably, time had no significant correlations with both absolute and relative abundance of ARGs (MRM, $P>0.05$ ). And compared with these important factors based on the PERMANOVA, we found these similar results based on the MRM analysis (Table S7). For the absolute abundance, treatment $\left(R^{2}=0.648\right)$, DO $\left(R^{2}=0.462\right)$ and intl1 (clinic) $\left(R^{2}=0.274\right)$ were three most important factors (MRM, all $\left.P=0.001\right)$. And for the relative abundance, treatment $\left(R^{2}=0.544\right)$, Do $\left(R^{2}\right.$ $=0.449)$ and $\mathrm{pH}\left(R^{2}=0.200\right)$ were three important factors $(\mathrm{MRM}$, all $P=0.001)$.

\subsection{Relationships among ARGs, water physicochemical factors, MGEs, and opportunistic pathogenic genera}

Firstly, we detected correlations between MGEs and ARGs (Fig.7-a, Fig.S12, Fig.S13-a). The total ARGs' absolute abundance was in positively relation to that of MGEs $\left(R^{2}=0.445, P<0.001\right)$ while there was no association between the total relative abundance of MGEs and ARGs (Fig.S12, $\left.R^{2}=0.007, P>0.05\right)$. In addition, network analysis showed that tnpA-01 was positively associated with the absolute abundance of vanC-03 $(r=0.860)$, tetD-01 $(r=0.852)$ and $m p h A-01(r=0.874)$, and the relative abundance of $d f r A 12(r=0.762)$, tetL-02 $(r=0.710)$ and aadA-01 ( $r=0.631)$. In addition, tnpA-07 was positively associated with the absolute abundance of $\mathrm{cm} / A 1-01(r=0.821)$, tetE $(r=0.820)$ and $\operatorname{aadA-01}(r=0.767)$, and the relative abundance of $d f r A 12(r=0.703)$ and tetL-02 ( $r=0.722)($ Fig.S13-a).

In addition, we found correlations between environmental factors and ARGs (Fig.6-a, b, Fig.S10). For instance, DO was negatively associated with tetracycline (tetB-01 and tetG-02) of both the absolute and relative abundance at three time points (Fig.S9, $P<0.01)$. In addition, CON was positively correlated with aminoglycoside (aadA-01) and tetracycline (tetB-01 and tetA-02) of both the absolute and relative abundance (Fig.6$\mathrm{a}, \mathrm{b}, P<0.01)$. And these correlations only existed at 7th and 15th day $(P<0.01)$, but were insignificant at 100th day (Fig.S10, $P>0.05)$. Similarly, network analysis showed the absolute abundance of aadA-01 ( $r=-0.854)$, tetB-01 $(r=-0.789)$ and tetA-02 ( $r=-0.787)$, and the relative abundance of tetB-01 ( $r=-0.831)$, aadA-01 $(r=-0.771)$ and $d f r A 12(r=-0.740)$ were negatively associated with DO. CON was positively related to the absolute abundance of aadA-01 $(r=0.857)$, tetB-01 $(r=0.779)$ and tetA-02 $(r=0.819)$, and the relative abundance of tetB-01 $(r=$ 0.865).

In addition, we also found the relative abundance of key genera (mean relative abundance $>1 \%$ ) was associated with the abundance of ARGs (Fig.6-c, d, Fig.7-b, Fig.S10, Fig.S13-b). Opportunistic pathogen like Comamonas was in positive correlation with absolute abundance of

Page $7 / 21$ 
tetD-01 $(r=0.895)$, catB8 $(r=0.861)$ and floR $(r=0.853)$, and the relative abundance of tetD-01 $(r=0.897), d f r A 12(r=0.881)$ and $\operatorname{catB} 8(r$ $=0.844)$ (Fig.S11). And Brevundimonas was positively associated with the absolute abundance of folA $(r=0.929)$, tetE $(r=0.865)$ and $v a n C$ $03(r=0.786)$, and the relative abundance of catB8 $(r=0.839)$ (Fig.S11).

\section{Discussion}

In this study, we used a fish model to explore the temporal variations of ARGs, bacterial communities and environmental factors when cadaver decomposes. Although our previous study has focused on the variation of microbial community during corpse decay of small volume water environment (Zhou et al., 2021), there is still no study regarding the ARGs and microbial community during corpse degradation in large-volume water systems at long time scale. However, studying the changes of ARGs and microbiome in large-volume water systems at long time period is essential and important to effectively assess hazards caused by corpse and provide more completely information for further investigates. Therefore, we used large-volume (i.e. 50L drinking water) in long time scale (i.e. 100 days) to imitate natural environment for studying the change and persistence of ARGs caused by carcass decomposition. In addition, we try to investigate the influencing factors related to ARGs during corpse degradation in order to provide suggestions and strategies for reducing the transmission and load of ARGs in drinking water.

\subsection{Carcass decay changes environmental properties, and thus affects the resistomes}

Corpse decay in the large-volume drinking water system significantly changed water chemistries was observed. CON significantly increased but DO slightly decreased over time, which is lined with previous researches (He et al., 2019; Xia et al., 2017). It is reported that CON is an indicator of total dissolved metal concentration in water environment (Yang et al., 2019; Zhang et al., 2019), which is likely to contribute to the variation of resistomes during carcass decomposition. Meanwhile, we found $\mathrm{NH}_{4}-\mathrm{N}$ was higher in experimental groups and gradually increased over time compared with control groups. On the contrary, $\mathrm{NO}_{3}-\mathrm{N}$ increased at the start while gradually decreased thereafter during corpse decomposition, in line with previous results (Metcalf et al., 2016; Yu et al., 2020). So we supposed carcass produces amounts of ammonia, and it transformed into nitrogen source under anaerobic environment. Moreover, TOC in experimental groups increased significantly, which was reasonable because cadaver decomposition may produce large quantities of organic matters (Ellingsen and Mohr, 1987; von der Lühe et al., 2018).

However, the $\mathrm{pH}$ range in both experimental and control groups was narrow (i.e. 6.97-7.98) and remain a slightly alkaline environment, which was inconsistent with previous study (Zhou et al., 2021). And our results showed that the pH value in experimental groups was lower than control groups at each time points. That may be because microbial fermentation produced organic acids and ammonia nitrogen during corpse decomposition, and then neutralized the alkalinity of the water. In addition, the pH value in experimental groups at 7th and 15th day was near neutral environment (i.e. 6.97-7.32), which can adapt to the survival of more microorganisms. And then, over corpse degradation, the organic acids were gradually utilized by certain microbes and the water environment is close to alkalinity at 100th day (i.e. 7.3-7.98). Hence, we can take these indicators (i.e. $\mathrm{NH}_{4}-\mathrm{N}, \mathrm{NO}_{3}-\mathrm{N}, \mathrm{TOC}$ ) to estimate the polluted situation of corpse in order to offer the appropriate solution.

Meanwhile, we also found that DO, $\mathrm{pH}$ and $\mathrm{CON}$ were three most important factors affecting ARGs. It has also been reported that DO and pH had significant effect on the abundance of ARGs (Tong et al., 2020). These environmental factors also are drivers in shaping microbial community (Yang et al., 2019; Zhou et al., 2021). Of note, we found environmental factors were also strongly associated with ARGs, which is in line with previous study (Pan et al., 2020). Therefore, it is likely very effective by regulating physicochemical factors (like adding alkali) for controlling the transmission of ARGs in drinking water and aquaculture environment.

\subsection{Carcass decomposition changes the microbiome and increases the abundance of opportunistic pathogens}

Similarly, our results demonstrated the temporal succession of microbial communities during corpse decomposition. We found that fish decomposing process existed a unique decomposer succession, which is consistent with previous study (Lobb et al., 2020). We also found Aeromonadaceae (UG), Nevskia and Azospira in experimental groups were the dominant genera at 7th, 15th and 100th day of fish decomposition, and were the dominant microbes in corpse groups for different decomposition stages separately. And Aeromonadaceae (UG), belonging to Aeromonadaceae-family, is known as a ubiquitous bacterium that exist in fish (Hossain et al., 2019), thus Aeromonadaceae (UG) may take part in the decomposition of submerged fresh. In addition, Nevskia, one of the dominant genera in drinking water system (KeinänenToivola et al., 2006), is likely to play a role in the floating decay stage of decomposition. Azospira is one of the nitrogen-fixing microorganism (Park et al., 2020). So we suppose that Azospira may be associated with the decrease of $\mathrm{NO}_{3}-\mathrm{N}$ in experimental groups. In addition, $B / v i i 28$ was first detected during corpse decomposition. It is reported that dominant decomposers of dissolved organic matter (Zhang et al., 2018), and thus Blvii28 may probably participate in the decomposition of organic matter during corpse decomposition.

Page $8 / 21$ 
Meanwhile, our data showed alpha diversity (i.e. chao 1 and observed OTUs) in the experimental groups gradually decreased over time. That may be associated with the alkalified water, and the consumption of oxygen and organic matters (i.e. TOC, TN). In addition, our data showed that corpse groups harbored higher abundance of 12 opportunistic bacteria such as Burkholderia, Legionella and Halomonas than control groups. For example, Burkholderia can cause a series of serious, often fatal, respiratory diseases in human (David et al., 2015). Of the Legionella species, most are considered pathogenic, and L. pneumophila is the leading cause of the severe pneumonia worldwide (Berjeaud et al., 2016). Halomonas, a newly discovered human pathogen, can cause infection and contamination in dialysis center and lead to bacteremia (Stevens et al., 2009). Corpse induced transfer of opportunistic pathogens to drinking water system may constitute potential risks for human health.

\subsection{Water resistomes increase due to corpse decomposition and have existed persistently}

Notably, our results showed abundance of MGEs and ARGs in experimental groups significantly increased. On the one hand, due to the extensive use of antibiotics in aquaculture (Cabello, 2006), ARGs can be transferred to from the skin, intestines and feces of dead farmed fish to water environment. On the other hand, it is reported that the spread of ARGs is closely associated with MGEs (Yang et al., 2020). And our results also showed that the ARGs' abundance was significantly related to MGEs. Thus, the enrichment of MGEs may promote the spread of ARGs, and lead to the increase of ARGs in water environment.

In addition, our data also indicated the types and abundance of most ARGs kept almost unchanged, and existed persistently within 100 days of corpse decomposition. And it is reported that ARGs is likely to persist in environment (Gupta et al., 2018). Therefore, we assumed these ARGs brought by corpse may be relatively stable and difficult to eliminate or reduce. The above results supported that the ARGs due to corpse decomposition in such a large-volume drinking water system still persisted at least 100 days.

\subsection{Opportunistic pathogens carrying MGEs may increase the spread of antibiotic resistomes}

It is widely known that MGEs can help ARGs to spread via HGT in different environments (Yu et al., 2020). Our result showed the total absolute abundance of ARGs was significantly related to MGEs. For example, integron like intl-1(clinic) has been proved as cassettes of more than 80 ARGs (Mazel, 2006). And our study also found that intl-1 (clinic) was related to most ARGs' absolute abundance (tetA-02, floR, $m p h A-01)$, which supports above study. Furthermore, we found that transposase like tnpA-01 was positively consistent with most of ARGs (like vanC-03, cm/A1-01 and tetD-01). It indicated that transposases may make contribution to spread and enrichment of resistomes, consistent with previous researches (Karkman et al., 2016; Yan et al., 2018).

In addition, we observed some opportunistic pathogens such as Brevundimonas, main cause of bacteraemia (Ryan and Pembroke, 2018), increased in experimental groups. Thus we can suppose Brevundimonas may be the potential host of ARGs during fish corpse decomposition. It is reported that Brevundimonas is significantly associated with tetracycline (Marti et al., 2013). The results in this study also implied that Brevundimonas was associated with tetB-01, tetE, tetQ. As a result, we can hypothesise ARG-host pathogens, can cause a greater hidden danger to the outbreaks and epidemics of water-borne diseases, if water source is polluted by corpses.

As we all known, the protection and management of drinking water sources is a vital issue for human health (Pandit and Kumar, 2015). For example, there is serous lack for management of drinking water and sanitation in Africa, especially in developing countries (Kasozi et al., 2019). Additionally, it is reported that water environment are identified as an ideal environment for dissemination of ARGs, and pose an additional health risk (Amarasiri et al., 2020). So our study is significant and necessary for drinking water security, to a certain extent. And compared with the small-volume water system's research (Zhou et al., 2021), studying the ARG profiles in the large-volume drinking water system is closer to the natural condition, and thus has greater practical significance. So we can provide some valuable suggestions for the risk assessment and management of polluted drinking water caused by corpse decay.

\section{Conclusion}

This study revealed except time, seven environmental factors, 12 opportunistic pathogens and corpse cay all significantly influenced the abundance of ARGs and ARG profile in the large-volume drinking water during a long time. Corpse decay increased the diversity and abundance of resistomes in large-volume drinking water within 100th days. In addition, environmental change (i.e. corpse decay) may selectively increase certain specific opportunistic bacteria which carried certain ARGs or MGEs, thus increased the spread of resistomes. Until the 100th day of decomposition, most of these ARGs still existed in the drinking water, and water system itself cannot eliminate ARGs, which indicated the persistence and indecomposability of ARGs. Thus, it is important to treat animal corpse in time before entering water system, and design optimal water management strategy to reduce the spread and enrichment of ARGs during corpse decomposition. 


\section{Declarations}

\section{Ethical approval}

The animal processing in our study was approved by the Animal Welfare and Ethics Committee of Chengdu Institute of Biology, Chinese Academy of Sciences (CIB-201602-174). The related experimental methods and procedures strictly followed the related guidelines.

\section{Consent to participate}

We affirm that all authors have participated in the research work and are fully aware of ethical responsibilities.

\section{Consent to Publish}

We affirm that all authors have agreed for submission of the paper to ESPR and are fully aware of ethical responsibilities.

\section{Authors Contributions}

H.L., X.L, T.L and W.S. were responsible for conceptualization, data curation and formal analysis. H.L. and X.L. was responsible for funding acquisition, W.S. and Y.W finished the work of investigation, methodology, project administration and resources. H.L., X.L. and W.S. finished the work of software, supervision, validation, visualization, writing-original draft and Writing-review \& editing. All authors have contributed to writing-review \& editing.

\section{Funding}

This work was supported by the National Natural Science Foundation of China (42007026 and 22006133).

\section{Competing Interests}

The authors have no conflicting interest and are aware of ethical responsibilities.

\section{Availability of data and materials}

The original 16S rRNA gene sequence data in this study were deposited at the European Nucleotide Archive by accession number PRJEB42593 (http://www.ebi.ac.uk/ena/data/view/PRJEB42593).

\section{References}

1. Amarasiri, M., Sano, D. and Suzuki, S. 2020. Understanding human health risks caused by antibiotic resistant bacteria (ARB) and antibiotic resistance genes (ARG) in water environments: Current knowledge and questions to be answered. Critical reviews in environmental science and technology 50(19), 2016-2059.

2. Balakrishnan, N., Colton, T., Everitt, B., Piegorsch, W., Ruggeri, F. and Teugels, J.L. 2014. Wiley StatsRef: Statistics Reference Online II Permutational Multivariate Analysis of Variance (PERMANOVA). 1-15.

3. Baquero, F., Martínez, J.-L. and Cantón, R. 2008. Antibiotics and antibiotic resistance in water environments. Current opinion in biotechnology 19(3), 260-265.

4. Ben, Y., Hu, M., Zhang, X., Wu, S., Wong, M.H., Wang, M., Andrews, C.B. and Zheng, C. 2020. Efficient detection and assessment of human exposure to trace antibiotic residues in drinking water. Water Res 175, 115699

5. Berjeaud, J.M., Chevalier, S., Schlusselhuber, M., Portier, E., Loiseau, C., Aucher, W., Lesouhaitier, O. and Verdon, J. 2016. Legionella pneumophila: The Paradox of a Highly Sensitive Opportunistic Waterborne Pathogen Able to Persist in the Environment. Front Microbiol 7, 486.

6. Cabello, F.C. 2006. Heavy use of prophylactic antibiotics in aquaculture: a growing problem for human and animal health and for the environment. Environ Microbiol 8(7), 1137-1144.

7. Caporaso, J.G., Kuczynski, J., Stombaugh, J., Bittinger, K., Bushman, F.D., Costello, E.K., Fierer, N., Peña, A.G., Goodrich, J.K., Gordon, J.I., Huttley, G.A., Kelley, S.T., Knights, D., Koenig, J.E., Ley, R.E., Lozupone, C.A., McDonald, D., Muegge, B.D., Pirrung, M., Reeder, J., Sevinsky, J.R., Turnbaugh, P.J., Walters, W.A., Widmann, J., Yatsunenko, T., Zaneveld, J. and Knight, R. 2010. QIIME allows analysis of highthroughput community sequencing data. Nat Methods 7(5), 335-336.

8. Carter, D.O., Yellowlees, D. and Tibbett, M. 2007. Cadaver decomposition in terrestrial ecosystems. Naturwissenschaften 94(1), 12-24. 
9. Chen, J., Shi, Y., Cheng, D., Jin, Y., Hutchins, W. and Liu, J. 2019. Survey of pathogenic bacteria of biofilms in a metropolitan drinking water distribution system. FEMS Microbiol Lett 366(20).

10. Cherven, K. 2013. Network graph analysis and visualization with Gephi.

11. Conly, J. and Johnston, B. 2005. Natural Disasters, Corpses and the Risk of Infectious Diseases. The Canadian journal of infectious diseases \& medical microbiology = Journal canadien des maladies infectieuses et de la microbiologie médicale / AMMI Canada 16, 269270.

12. Cui, E.P., Gao, F., Liu, Y., Fan, X.Y., Li, Z.Y., Du, Z.J., Hu, C. and Neal, A.L. 2018. Amendment soil with biochar to control antibiotic resistance genes under unconventional water resources irrigation: Proceed with caution. Environmental Pollution 240, $475-484$.

13. Czekalski, N., Díez, E.G. and Bürgmann, H. 2014. Wastewater as a point source of antibiotic-resistance genes in the sediment of a freshwater lake. The ISME journal 8(7), 1381-1390.

14. David, J., Bell, R.E. and Clark, G.C. 2015. Mechanisms of Disease: Host-Pathogen Interactions between Burkholderia Species and Lung Epithelial Cells. Front Cell Infect Microbiol 5, 80.

15. Deng, Y., He, Z., Xiong, J., Yu, H., Xu, M., Hobbie, S.E., Reich, P.B., Schadt, C.W., Kent, A. and Pendall, E. 2016. Elevated carbon dioxide accelerates the spatial turnover of soil microbial communities. Global Change Biology 22(2).

16. Ellingsen, T.E. and Mohr, V. 1987. Biochemistry of the autolytic processes in Antarctic krill post mortem. Autoproteolysis. Biochem J 246(2), 295-305.

17. Emmons, A.L., DeBruyn, J.M., Mundorff, A.Z., Cobaugh, K.L. and Cabana, G.S. 2017. The persistence of human DNA in soil following surface decomposition. Sci Justice 57(5), 341-348.

18. Falkinham, J.O., Pruden, A. and Edwards, M. 2015. Opportunistic Premise Plumbing Pathogens: Increasingly Important Pathogens in Drinking Water. Pathogens 4(2), 373-386.

19. Fan, H. 2011. The Method of Improving the Accuracy of Determination of Total Nitrogen by Using the Potassium Persulfate UV Spectrophotometry. Journal of Guangxi Academy of Sciences.

20. Guo, J., Fu, X., Liao, H., Hu, Z., Long, L., Yan, W., Ding, Y., Zha, L., Guo, Y., Yan, J., Chang, Y. and Cai, J. 2016. Potential use of bacterial community succession for estimating post-mortem interval as revealed by high-throughput sequencing. Sci Rep 6, 24197.

21. Gupta, S.K., Shin, H., Han, D., Hur, H.G. and Unno, T. 2018. Metagenomic analysis reveals the prevalence and persistence of antibioticand heavy metal-resistance genes in wastewater treatment plant. J Microbiol 56(6), 408-415.

22. Han, Z., Zhang, Y., An, W., Lu, J., Hu, J. and Yang, M. 2020. Antibiotic resistomes in drinking water sources across a large geographical scale: Multiple drivers and co-occurrence with opportunistic bacterial pathogens. Water Research 183, 116088.

23. He, J., Guo, J., Fu, X. and Cai, J. 2019. Potential use of high-throughput sequencing of bacterial communities for postmortem submersion interval estimation. Braz J Microbiol 50(4), 999-1010.

24. Heimesaat, M.M., Boelke, S., Fischer, A., Haag, L.M., Loddenkemper, C., Kuhl, A.A., Gobel, U.B. and Bereswill, S. 2012. Comprehensive Postmortem Analyses of Intestinal Microbiota Changes and Bacterial Translocation in Human Flora Associated Mice. Plos One 7(7).

25. Hossain, S., De Silva, B.C.J., Wimalasena, S.H.M.P., Pathirana, H.N.K.S., Dahanayake, P.S. and Heo, G.-J. 2019. Characterization of Virulence Determinants And Multiple Antimicrobial Resistance Profiles in Motile Aeromonas Spp. Isolated from Ornamental Goldfish (Carassius Auratus). Journal of Exotic Pet Medicine 29, 51-62.

26. Hyde, E.R., Haarmann, D.P., Lynne, A.M., Bucheli, S.R. and Petrosino, J.F. 2013. The Living Dead: Bacterial Community Structure of a Cadaver at the Onset and End of the Bloat Stage of Decomposition. Plos One 8(10).

27. Karkman, A., Johnson, T.A., Lyra, C., Stedtfeld, R.D., Tamminen, M., Tiedje, J.M. and Virta, M. 2016. High-throughput quantification of antibiotic resistance genes from an urban wastewater treatment plant. Fems Microbiol Ecol 92(3).

28. Kasozi, K.I., Namubiru, S., Kamugisha, R., Eze, E.D., Tayebwa, D.S., Ssempijja, F., Okpanachi, A.O., Kinyi, H.W., Atusiimirwe, J.K., Suubo, J., Fernandez, E.M., Nshakira, N. and Tamale, A. 2019. Safety of Drinking Water from Primary Water Sources and Implications for the General Public in Uganda. J Environ Public Health 2019, 7813962.

29. Keinänen-Toivola, M.M., Revetta, R.P. and Santo Domingo, J.W. 2006. Identification of active bacterial communities in a model drinking water biofilm system using 16S rRNA-based clone libraries. FEMS Microbiol Lett 257(2), 182-188.

30. Li, H., Li, T., Beasley, D.E., Petr, H., Xiao, Z., Zhang, S., Li, J., Lin, Q. and Li, X. 2016. Diet Diversity Is Associated with Beta but not Alpha Diversity of Pika Gut Microbiota. Frontiers in Microbiology 7(758).

31. Li, K.F., Luo, Y.-k., Feng, Q.-c. and Yao, L. 2012. Effect of fish-scale protein hydrolysates-based films on preservation of crucian carp (Carassius auratus). Journal of Fisheries of China 35, 1113-1119.

32. Liu, L., Su, J.Q., Guo, Y., Wilkinson, D.M., Liu, Z., Zhu, Y.G. and Yang, J. 2018. Large-scale biogeographical patterns of bacterial antibiotic resistome in the waterbodies of China. Environment International 117(AUG.), 292.

Page $11 / 21$ 
33. Lobb, B., Hodgson, R., Lynch, M.D., Mansfield, M.J., Cheng, J., Charles, T.C., Neufeld, J.D., Craig, P.M. and Doxey, A.C. 2020. Time series resolution of the fish necrobiome reveals a decomposer succession involving toxigenic bacterial pathogens. MSystems $5(2)$.

34. Lozupone, C. and Knight, R. 2005. UniFrac: a new phylogenetic method for comparing microbial communities. Appl Environ Microbiol 71(12), 8228-8235.

35. Magoč, T. and Salzberg, S.L. 2011. FLASH: fast length adjustment of short reads to improve genome assemblies. Bioinformatics 27(21), 2957-2963.

36. Marti, E., Jofre, J. and Balcazar, J.L. 2013. Prevalence of antibiotic resistance genes and bacterial community composition in a river influenced by a wastewater treatment plant. PLoS One 8(10), e78906.

37. Marti, E., Variatza, E. and Balcazar, J.L. 2014. The role of aquatic ecosystems as reservoirs of antibiotic resistance. Trends in microbiology 22(1), 36-41.

38. Mazel, D. 2006. Integrons: agents of bacterial evolution. Nat Rev Microbiol 4(8), 608-620.

39. Metcalf, J.L., Xu, Z.Z., Weiss, S., Lax, S., Van Treuren, W., Hyde, E.R., Song, S.J., Amir, A., Larsen, P. and Sangwan, N. 2016. Microbial community assembly and metabolic function during mammalian corpse decomposition. Science 351(6269), $158-162$.

40. Ming-Xing, Y.U., Zheng, H.Y. and Wang, G. 2005. Discussion about Common Problems and Solution Methods in Determination of Ammoniacal Nitrogen by Nessler's Reagent Colorimetric Method. Arid Environmental Monitoring.

41. Ouyang, W.Y., Huang, F.Y., Zhao, Y., Li, H. and Su, J.Q. 2015. Increased levels of antibiotic resistance in urban stream of Jiulongjiang River, China. Appl Microbiol Biotechnol 99(13), 5697-5707.

42. Pan, X., Lin, L., Zhang, W., Dong, L. and Yang, Y. 2020. Metagenome sequencing to unveil the resistome in a deep subtropical lake on the Yunnan-Guizhou Plateau, China. Environ Pollut 263(Pt B), 114470.

43. Pandit, A.B. and Kumar, J.K. 2015. Clean Water for Developing Countries. Annu Rev Chem Biomol Eng 6, $217-246$.

44. Park, H.J., Kwon, J.H., Yun, J. and Cho, K.S. 2020. Characterization of nitrous oxide reduction by Azospira sp. HJ23 isolated from advanced wastewater treatment sludge. J Environ Sci Health A Tox Hazard Subst Environ Eng 55(12), $1459-1467$.

45. Parmenter, R.R. and MacMahon, J.A. 2009. Carrion decomposition and nutrient cycling in a semiarid shrub-steppe ecosystem. Ecological Monographs 79(4), 637-661.

46. Puente-Sánchez, F., Aguirre, J. and Parro, V. 2015. A novel conceptual approach to read-filtering in high-throughput amplicon sequencing studies. Nucleic Acids Research 44(4), e40-e40.

47. Qian, X., Gu, J., Sun, W., Wang, X.-J., Su, J.-Q. and Stedfeld, R. 2018. Diversity, abundance, and persistence of antibiotic resistance genes in various types of animal manure following industrial composting. Journal of Hazardous Materials 344, 716-722.

48. Rai, K.R., Mukhiya, R.K., Thapa, S., Rai, G., Kc, S., Thapa, P.M., Shrestha, P. and Rai, S.K. 2019. Diarrheal disease outbreak in Gaidatar village of Rautahat District, Nepal. BMC Res Notes 12(1), 124.

49. Ryan, M.P. and Pembroke, J.T. 2018. Brevundimonas spp: Emerging global opportunistic pathogens. Virulence 9(1), 480-493.

50. Sidhu, J.P.S., Gupta, V.V.S.R., Stange, C., Ho, J., Harris, N., Barry, K., Gonzalez, D., Van Nostrand, J.D., Zhou, J., Page, D., Tiehm, A. and Toze, S. 2020. Prevalence of antibiotic resistance and virulence genes in the biofilms from an aquifer recharged with stormwater. Water Research 185, 116269.

51. Singh, B., Minick, K.J., Strickland, M.S., Wickings, K.G., Crippen, T.L., Tarone, A.M., Benbow, M.E., Sufrin, N., Tomberlin, J.K. and Pechal, J.L. 2018. Temporal and spatial impact of human cadaver decomposition on soil bacterial and arthropod community structure and function. Frontiers in microbiology 8, 2616.

52. Spicka, A., Johnson, R., Bushing, J., Higley, L.G. and Carter, D.O. 2011. Carcass mass can influence rate of decomposition and release of ninhydrin-reactive nitrogen into gravesoil. Forensic Sci Int 209(1-3), 80-85.

53. Stevens, D.A., Hamilton, J.R., Johnson, N., Kim, K.K. and Lee, J.S. 2009. Halomonas, a newly recognized human pathogen causing infections and contamination in a dialysis center: three new species. Medicine (Baltimore) 88(4), 244-249.

54. Su, H., Hu, X., Xu, Y., Xu, W., Huang, X., Wen, G., Yang, K., Li, Z. and Cao, Y. 2018. Persistence and spatial variation of antibiotic resistance genes and bacterial populations change in reared shrimp in South China. Environ Int 119, 327-333.

55. Tang, X.J., Lou, C.L., Wang, S.X., Lu, Y.H., Liu, M., Hashmi, M.Z., Liang, X.Q., Li, Z.P., Liao, Y.L., Qin, W.J., Fan, F., Xu, J.M. and Brookes, P.C. 2015. Effects of long-term manure applications on the occurrence of antibiotics and antibiotic resistance genes (ARGs) in paddy soils: Evidence from four field experiments in south of China. Soil Biol Biochem 90, 179-187.

56. Tong, L., Qin, L., Guan, C., Wilson, M.E., Li, X., Cheng, D., Ma, J., Liu, H. and Gong, F. 2020. Antibiotic resistance gene profiling in response to antibiotic usage and environmental factors in the surface water and groundwater of Honghu Lake, China. Environ Sci Pollut Res Int 27(25), 31995-32005. 
57. Visco, G., Campanella, L. and Nobili, V. 2005. Organic carbons and TOC in waters: an overview of the international norm for its measurements. Microchemical Journal 79(1-2), 185-191.

58. von der Lühe, B., Birk, J.J., Dawson, L., Mayes, R.W. and Fiedler, S. 2018. Steroid fingerprints: Efficient biomarkers of human decomposition fluids in soil. Organic Geochemistry 124, 228-237.

59. Wang, F.H., Qiao, M., Su, J.Q., Chen, Z., Zhou, X. and Zhu, Y.G. 2014. High throughput profiling of antibiotic resistance genes in urban park soils with reclaimed water irrigation. Environ Sci Technol 48(16), 9079-9085.

60. Wang, X., Han, J., Xu, L., Gao, J. and Zhang, Q. 2011. Effects of anthropogenic activities on chemical contamination within the Grand Canal, China. Environ Monit Assess 177(1-4), 127-139.

61. Weiss, S., Carter, D.O., Metcalf, J.L. and Knight, R. 2016. Carcass mass has little influence on the structure of gravesoil microbial communities. Int J Legal Med 130(1), 253-263.

62. Wright, G.D. and Poinar, H. 2012. Antibiotic resistance is ancient: implications for drug discovery. Trends Microbiol 20(4), $157-159$.

63. Xia, Z.Y., Zhai, X.D., Liu, B.B., Zheng, Z., Zhao, L.L. and Mo, Y.N. 2017. [Relationship between Electrical Conductivity and Decomposition Rate of Rat Postmortem Skeletal Muscle]. Fa Yi Xue Za Zhi 33(1), 17-20.

64. Yan, W., Guo, Y., Xiao, Y., Wang, S., Ding, R., Jiang, J., Gang, H., Wang, H., Yang, J. and Zhao, F. 2018. The changes of bacterial communities and antibiotic resistance genes in microbial fuel cells during long-term oxytetracycline processing. Water Res $142,105-114$.

65. Yang, F., Han, B., Gu, Y. and Zhang, K. 2020. Swine liquid manure: a hotspot of mobile genetic elements and antibiotic resistance genes. Sci Rep 10(1), 15037.

66. Yang, Y., Liu, G., Ye, C. and Liu, W. 2019. Bacterial community and climate change implication affected the diversity and abundance of antibiotic resistance genes in wetlands on the Qinghai-Tibetan Plateau. J Hazard Mater 361, 283-293.

67. Yang, Y., Song, W., Lin, H., Wang, W., Du, L. and Xing, W. 2018. Antibiotics and antibiotic resistance genes in global lakes: A review and meta-analysis. Environment International 116, 60-73.

68. Young, L.S., Stevens, P. and Kaijser, B. 1982. Gram-negative pathogens in septicaemic infections. Scand J Infect Dis Suppl 31 , 78-94.

69. Yu, Q., Zhou, R., Wang, Y., Feng, T. and Li, H. 2020. Corpse decomposition increases nitrogen pollution and alters the succession of nirKtype denitrifying communities in different water types. Science of The Total Environment 747, 141472.

70. Zhang, L., Zhang, S., Lv, X., Qiu, Z., Zhang, Z. and Yan, L. 2018. Dissolved organic matter release in overlying water and bacterial community shifts in biofilm during the decomposition of Myriophyllum verticillatum. Sci Total Environ 633, $929-937$.

71. Zhang, X., Johnston, E.R., Liu, W., Li, L. and Han, X. 2016. Environmental changes affect the assembly of soil bacterial community primarily by mediating stochastic processes. Glob Chang Biol 22(1), 198-207.

72. Zhang, Y.J., Hu, H.W., Yan, H., Wang, J.T., Lam, S.K., Chen, Q.L., Chen, D. and He, J.Z. 2019. Salinity as a predominant factor modulating the distribution patterns of antibiotic resistance genes in ocean and river beach soils. Sci Total Environ 668, 193-203.

73. Zhou, R., Wang, Y., Hilal, M.G., Yu, Q., Feng, T. and Li, H. 2021. Temporal succession of water microbiomes and resistomes during carcass decomposition in a fish model. J Hazard Mater 403, 123795.

74. Zhu, L., Wang, X., Wang, K., Yang, Q., He, J., Qin, Z., Geng, Y., Ouyang, P. and Huang, X. 2017. Outbreak of infectious pancreatic necrosis virus (IPNV) in farmed rainbow trout in China. Acta tropica 170, 63-69.

75. Zimmerman, K.A. and Wallace, J.R. 2008. The potential to determine a postmortem submersion interval based on algal/diatom diversity on decomposing mammalian carcasses in brackish ponds in Delaware. J Forensic Sci 53(4), 935-941.

\section{Tables}

Table 1. Two-way ANOVA showing the effects of time and treatment on the classification of ARGs in drinking water system.

Bold font means the significance at $P<0.05$ level.

Table 2. PERMANOVA analysis showing the effects of treatment, time, environmental factors and MGEs on ARGs across all samples. 


\begin{tabular}{|c|c|c|c|c|c|c|c|c|c|c|c|c|}
\hline \multirow{3}{*}{$\begin{array}{l}\text { Two-way ANOVA } \\
\text { Factors }\end{array}$} & \multicolumn{6}{|c|}{ Absolute abundance } & \multicolumn{6}{|c|}{ Relative abundance } \\
\hline & \multicolumn{2}{|c|}{ Treatment } & \multicolumn{2}{|l|}{ Time } & \multicolumn{2}{|c|}{ Treatment*Time } & \multicolumn{2}{|c|}{ Treatment } & \multicolumn{2}{|l|}{ Time } & \multicolumn{2}{|c|}{ Treatment* ${ }^{\star}$ Time } \\
\hline & $\mathrm{F}$ & $P$ & $\mathrm{~F}$ & $P$ & $\mathrm{~F}$ & $P$ & $\mathrm{~F}$ & $P$ & $\mathrm{~F}$ & $P$ & $\mathrm{~F}$ & $P$ \\
\hline Aminoglycoside & 36.402 & $<0.001$ & 0.714 & 0.500 & 0.713 & 0.500 & 10.360 & 0.004 & 6.122 & 0.007 & 2.229 & 0.129 \\
\hline Beta-Lactamase & 19.872 & $<0.001$ & 5.762 & 0.009 & 5.771 & 0.009 & 1.842 & 0.187 & 5.762 & 0.009 & 6.014 & 0.008 \\
\hline Chloramphenicol & 86.895 & $<0.001$ & 16.181 & $<0.001$ & 16.212 & $<0.001$ & 0.316 & 0.579 & 1.140 & 0.336 & 4.386 & 0.024 \\
\hline MLSB & 39.121 & $<0.001$ & 17.684 & $<0.001$ & 17.693 & $<0.001$ & 0.130 & 0.721 & 0.532 & 0.594 & 6.950 & 0.004 \\
\hline Multidrug & 154.190 & $<0.001$ & 21.787 & $<0.001$ & 21.785 & $<0.001$ & 1.705 & 0.204 & 5.610 & 0.010 & 1.390 & 0.268 \\
\hline Sulfonamide & 17.652 & $<0.001$ & 6.068 & 0.007 & 6.068 & 0.007 & 10.719 & 0.003 & 4.014 & 0.031 & 0.771 & 0.474 \\
\hline Tetracycline & 61.882 & $<0.001$ & 5.222 & 0.013 & 5.222 & 0.013 & 70.181 & $<0.001$ & 4.676 & 0.019 & 4.405 & 0.023 \\
\hline Vancomycin & 83.833 & $<0.001$ & 9.740 & 0.001 & 9.781 & 0.001 & 5.497 & 0.028 & 5.869 & 0.008 & 4.406 & 0.023 \\
\hline MGEs & 86.738 & $<0.001$ & 0.848 & 0.441 & 0.852 & 0.439 & 0.046 & 0.832 & 1.783 & 0.190 & 1.321 & 0.285 \\
\hline Others & 14.722 & 0.001 & 2.246 & 0.128 & 2.246 & 0.128 & 3.339 & 0.080 & 0.398 & 0.676 & 0.603 & 0.555 \\
\hline
\end{tabular}

\begin{tabular}{|lllllll|}
\hline PERMANOVA & \multicolumn{3}{l}{ Absolute abundance } & \multicolumn{3}{l|}{ Relative abundance } \\
\cline { 2 - 7 } & $\mathrm{F}$ & $R 2$ & $P$ & $\mathrm{~F}$ & $R 2$ & $P$ \\
\hline Time & 1.0101 & 0.348 & 0.373 & 1.5898 & 0.054 & 0.147 \\
\hline Treatment & 15.951 & 0.363 & $\mathbf{0 . 0 0 1}$ & 20.929 & 0.428 & $\mathbf{0 . 0 0 1}$ \\
\hline Alpha diversity & 2.007 & 0.067 & 0.072 & 2.5382 & 0.083 & $\mathbf{0 . 0 6 0}$ \\
\hline Beta diversity & 8.7929 & 0.239 & $\mathbf{0 . 0 0 1}$ & 11.286 & 0.287 & $\mathbf{0 . 0 0 1}$ \\
\hline pH & 10.105 & 0.265 & $\mathbf{0 . 0 0 1}$ & 13.008 & 0.317 & $\mathbf{0 . 0 0 1}$ \\
\hline Conductivity(us) & 8.1452 & 0.225 & $\mathbf{0 . 0 0 1}$ & 11.888 & 0.298 & $\mathbf{0 . 0 0 1}$ \\
\hline DO(mg/L) & 15.689 & 0.359 & $\mathbf{0 . 0 0 1}$ & 20.735 & 0.425 & $\mathbf{0 . 0 0 1}$ \\
\hline NH4-N(mg/kg) & 6.2406 & 0.182 & $\mathbf{0 . 0 0 1}$ & 9.0178 & 0.244 & $\mathbf{0 . 0 0 1}$ \\
\hline NO3-N(mg/kg) & 3.6632 & 0.116 & $\mathbf{0 . 0 0 4}$ & 5.1083 & 0.154 & $\mathbf{0 . 0 0 2}$ \\
\hline TOC(\%) & 5.382 & 0.161 & $\mathbf{0 . 0 0 2}$ & 5.9372 & 0.175 & $\mathbf{0 . 0 0 3}$ \\
\hline TN(\%) & 7.1852 & 0.204 & $\mathbf{0 . 0 0 1}$ & 7.8095 & 0.218 & $\mathbf{0 . 0 0 2}$ \\
\hline cIntl-1(class1) & 1.2347 & 0.042 & 0.322 & 3.7186 & 0.117 & $\mathbf{0 . 0 1 2}$ \\
\hline intl-1(clinic) & 10.217 & 0.267 & $\mathbf{0 . 0 0 1}$ & 5.2197 & 0.157 & $\mathbf{0 . 0 0 3}$ \\
\hline tnpA-01 & 5.7393 & 0.170 & $\mathbf{0 . 0 0 1}$ & 3.8261 & 0.120 & $\mathbf{0 . 0 0 1}$ \\
\hline tnpA-02 & 2.0715 & 0.069 & $\mathbf{0 . 0 3 8}$ & 0.77055 & 0.027 & 0.491 \\
\hline tnpA-04 & 6.9423 & 0.199 & $\mathbf{0 . 0 0 1}$ & 0.90123 & 0.031 & 0.583 \\
\hline tnpA-05 & 5.6926 & 0.169 & $\mathbf{0 . 0 0 1}$ & 3.7184 & 0.117 & $\mathbf{0 . 0 0 7}$ \\
\hline tnpA-07 & 4.3242 & 0.134 & $\mathbf{0 . 0 0 1}$ & 2.4713 & 0.081 & 0.071 \\
\hline IS613 & 0.96387 & 0.033 & 0.554 & 1.4345 & 0.049 & 0.103 \\
\hline
\end{tabular}

Abbreviations: Alpha diversity, Observed species (OTUs); Beta diversity, PCOA1; DO, dissolve oxygen; $\mathrm{NH}_{4}-\mathrm{N}$, ammonia nitrogen; $\mathrm{NO}_{3}-\mathrm{N}$, nitrate$\mathrm{N}$; TOC, total organic carbon; TN, total nitrogen.

Bold font means the significance at $P<0.05$ level. 

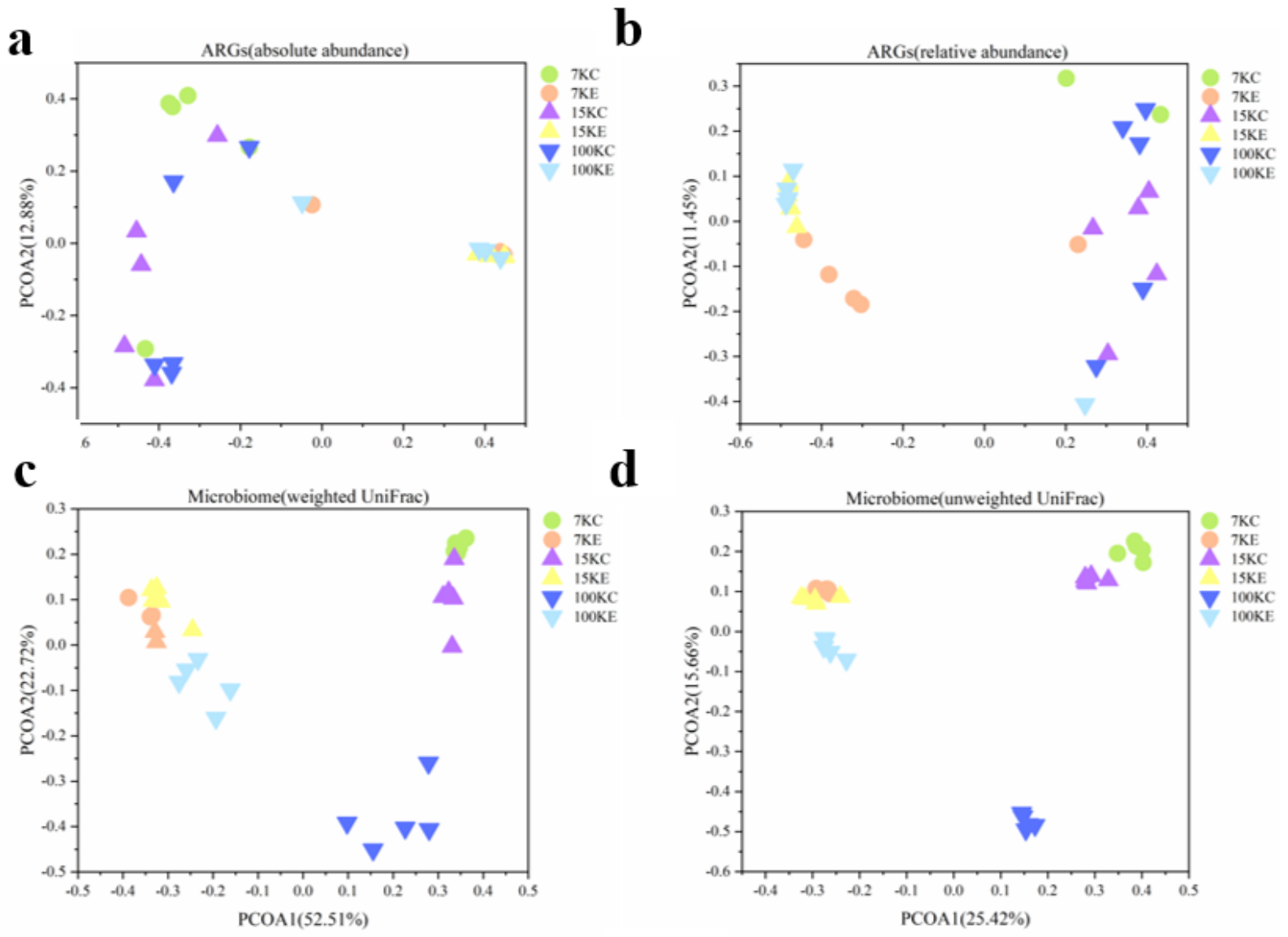

\section{Figure 1}

Principal coordinate analysis (PCoA) analysis showing the bray-curits of absolute abundance (a) and relative abundance (b) and the bacterial community (c-d) structures among different time points. Principal coordinate analysis (PCoA) analysis plots based on the weighted and unweighted UniFrac distance matrix in different time points. Abbreviations: $7 \mathrm{KC}, 15 \mathrm{KC}$ and $100 \mathrm{KC}$, control groups at 7 th day, 15 th day and 100th day during corpse decomposition; 7KE, 15KE and 100KE, experimental groups at 7th day, 15th day and 100th day during corpse decomposition. 
$\mathbf{a}$

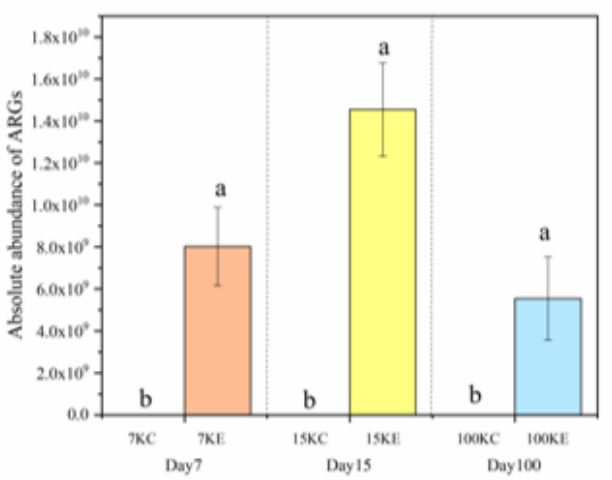

C

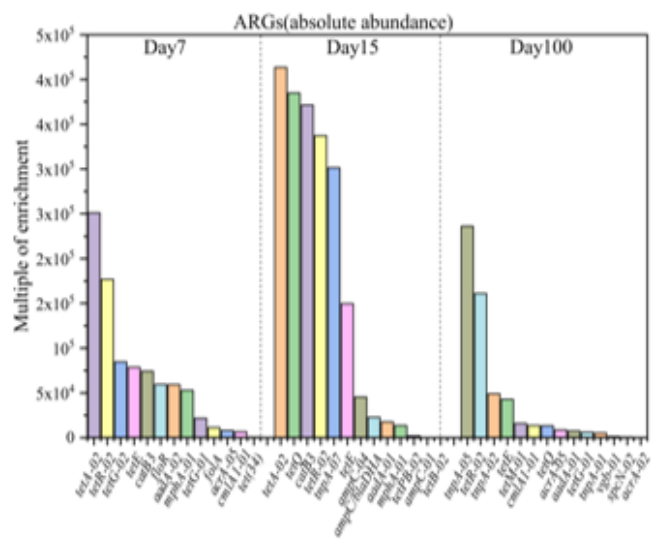

b

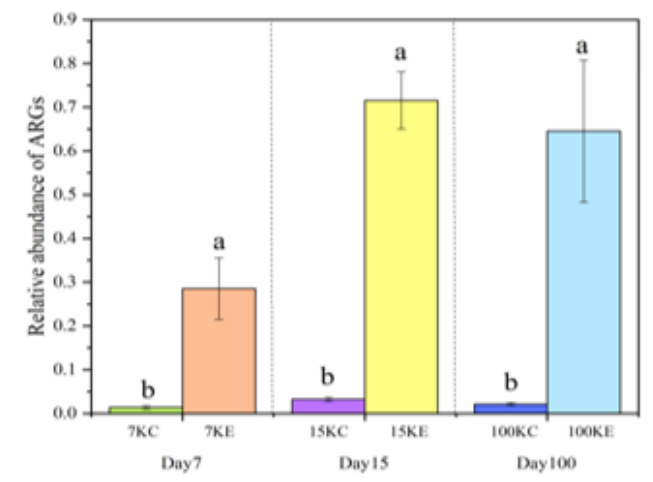

d

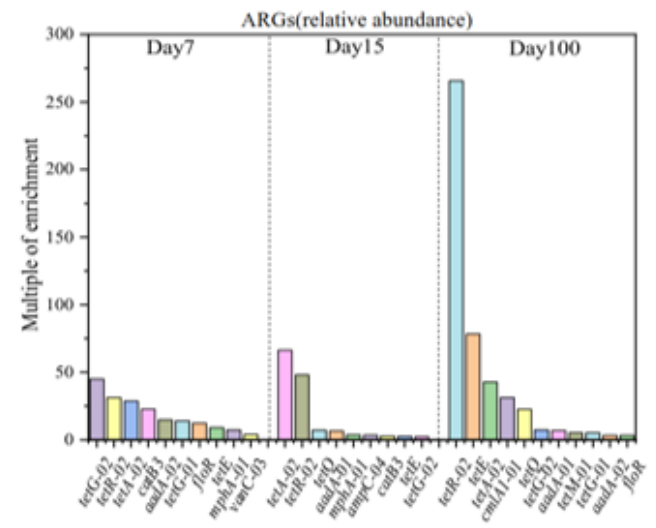

\section{Figure 2}

The shifts of absolute and relative abundance of total ARGs from water bacterial communities in all samples between the experimental and control groups in samples $(a, b)$. The multiple of enrichment of absolute and relative abundance of different types of antibiotic resistance genes (ARGs) gene in three experimental groups at each time point (c, d). Abbreviations: 7KC, $15 \mathrm{KC}$ and $100 \mathrm{KC}$, control groups at 7th day, 15th day and 100th day during corpse decomposition; 7KE, 15KE and 100KE, experimental groups at 7th day, 15th day and 100th day during corpse decomposition. 


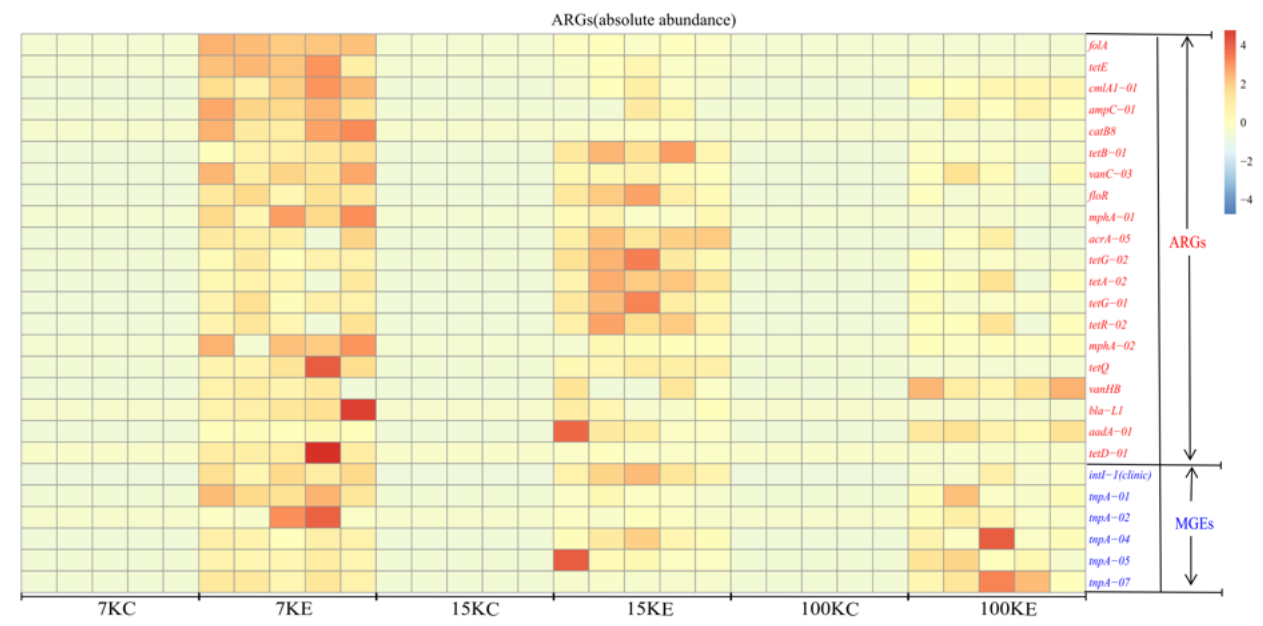

\section{b}

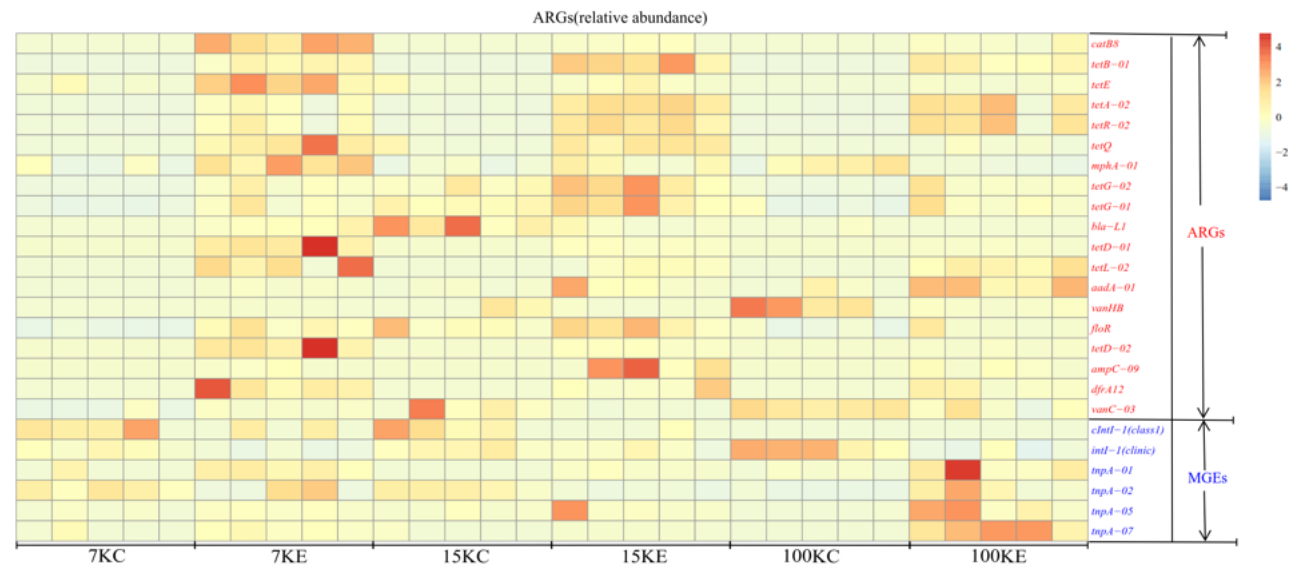

Figure 3

Heatmap showing the change of absolute and relative abundance after screening out the significant genes $(a, b)$. Abbreviations: 7KC, 15KC and $100 \mathrm{KC}$, control groups at 7th day, 15th day and 100th day during corpse decomposition; 7KE, 15KE and 100KE, experimental groups at 7th day, 15th day and 100th day during corpse decomposition. 
a

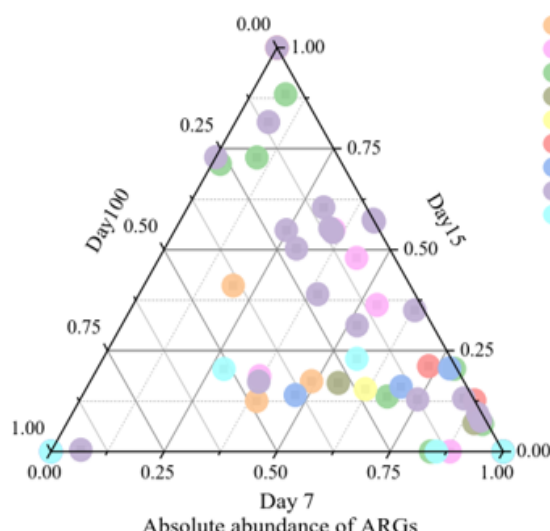

c

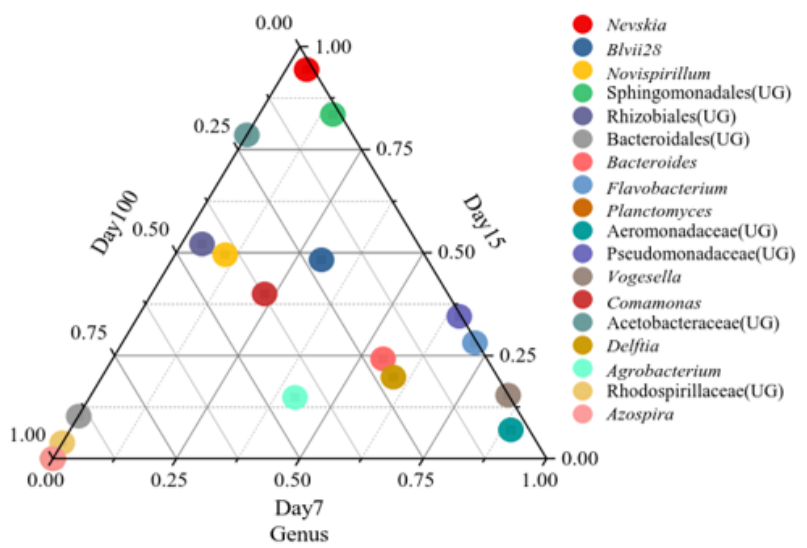

Absolute abundance of ARGs

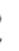

b Aminoglycosid
Multidrug Beta_Lactamase

Others

Chloramphenicol

Sulfonamide

MLSB

Tetracycline

Vancomycin

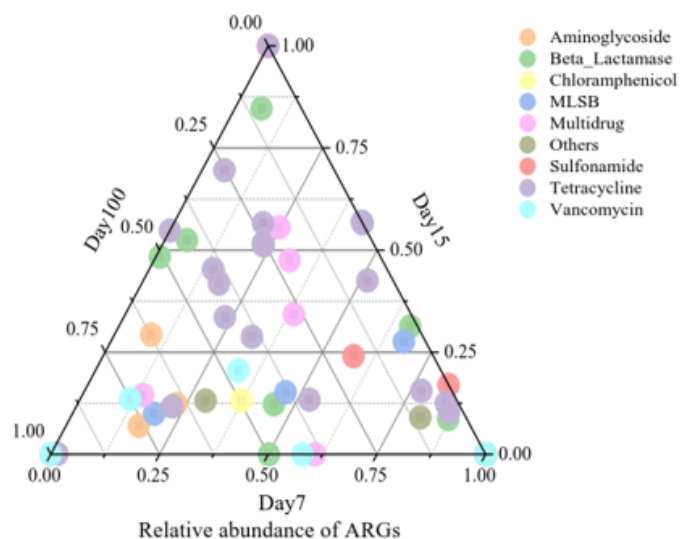

Relative abundance of ARGs

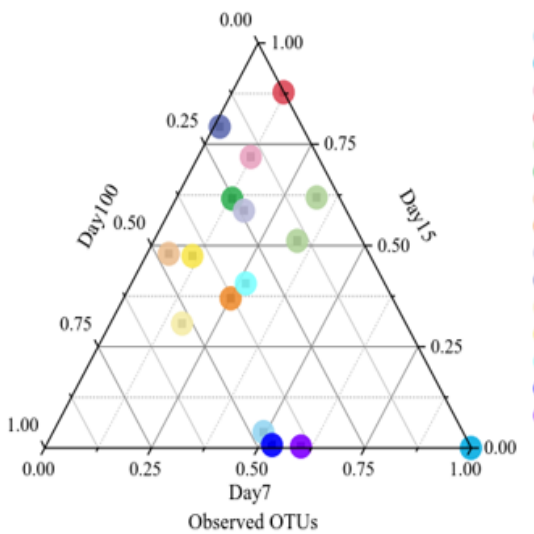

OTU32479_Nevskia ramosa

OTU31089 Acetobacteraceae(UG)

OTU41625 Aeromonadaceae(UG)

OTU3597_Azospira(UG)

OTU35264_Bacteroidales(UG)

OTU54937_Bacteroides(UG)

OTU27272 Blvii28(UG)

OTU53746_Comamonas(UG)

OTU25782_Delftia(UG)

OTU36631 Flavobacterium(UG)

OTU48291 mitochondria

OTU46116_Novispirillum(UG)

OTU19506_Pseudomonadaceae

OTU51246_Rhizobiales(UG)

OTU32481_Sphingomonadales(UG)

\section{Figure 4}

The classification's distribution of absolute abundance and relative abundance of total ARGs in experimental groups (a, b). Bacterial community composition of three experimental groups at the genus level (c) and the OTUs level (d) during different stages of carcass decomposition. 
$\mathbf{a}$

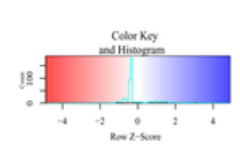

c
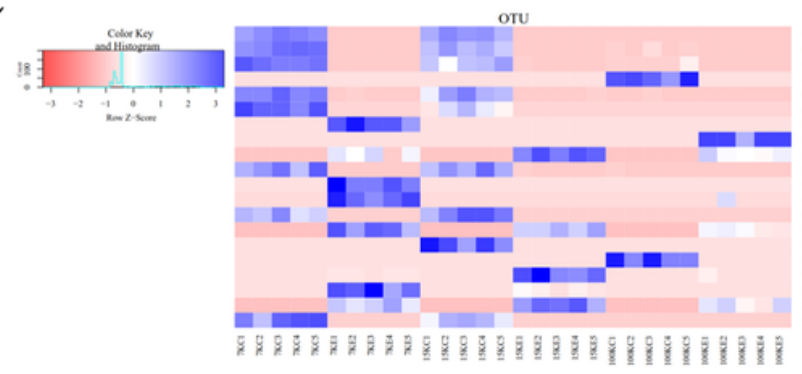

b

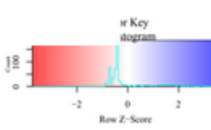

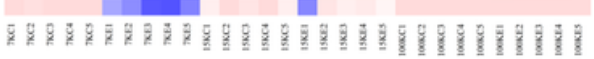

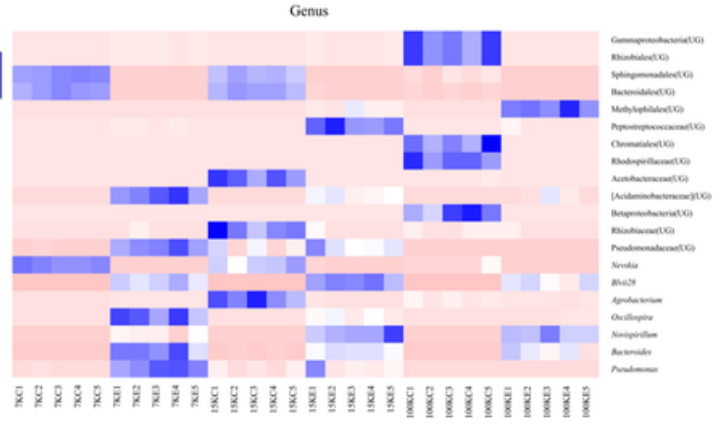

d
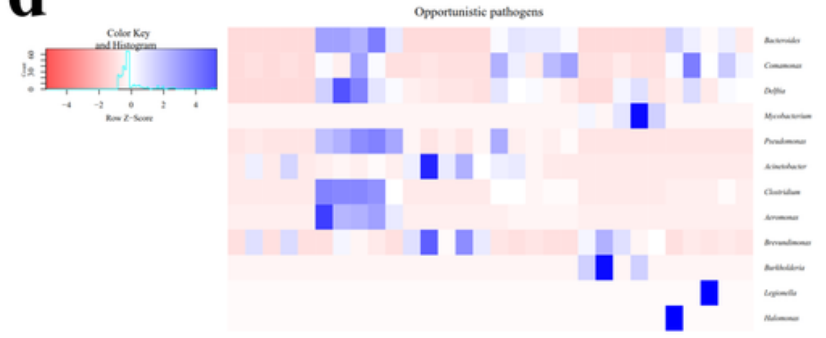

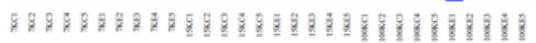

\section{Figure 5}

Heatmap showing the variation of the phyla (a), genera (b), the OTU (c) and opportunistic pathogenic genera (d) at each time point during corpse decomposition. 

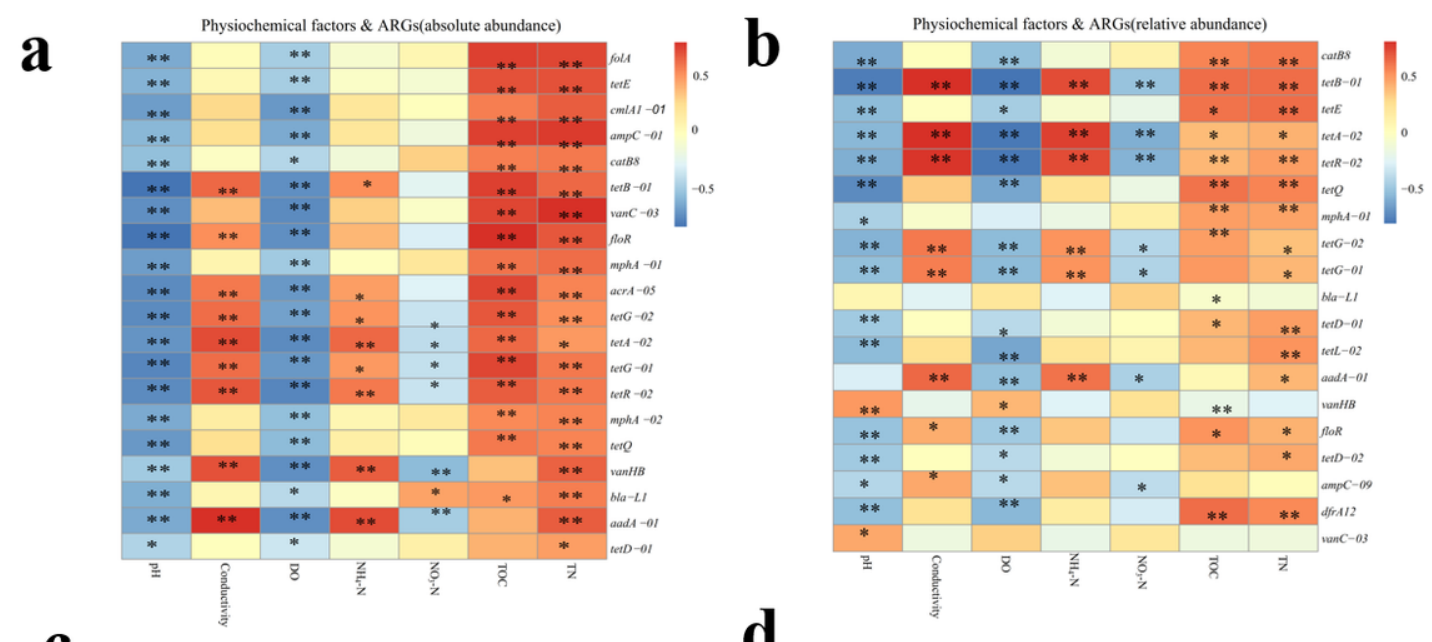

$\mathbf{C}$
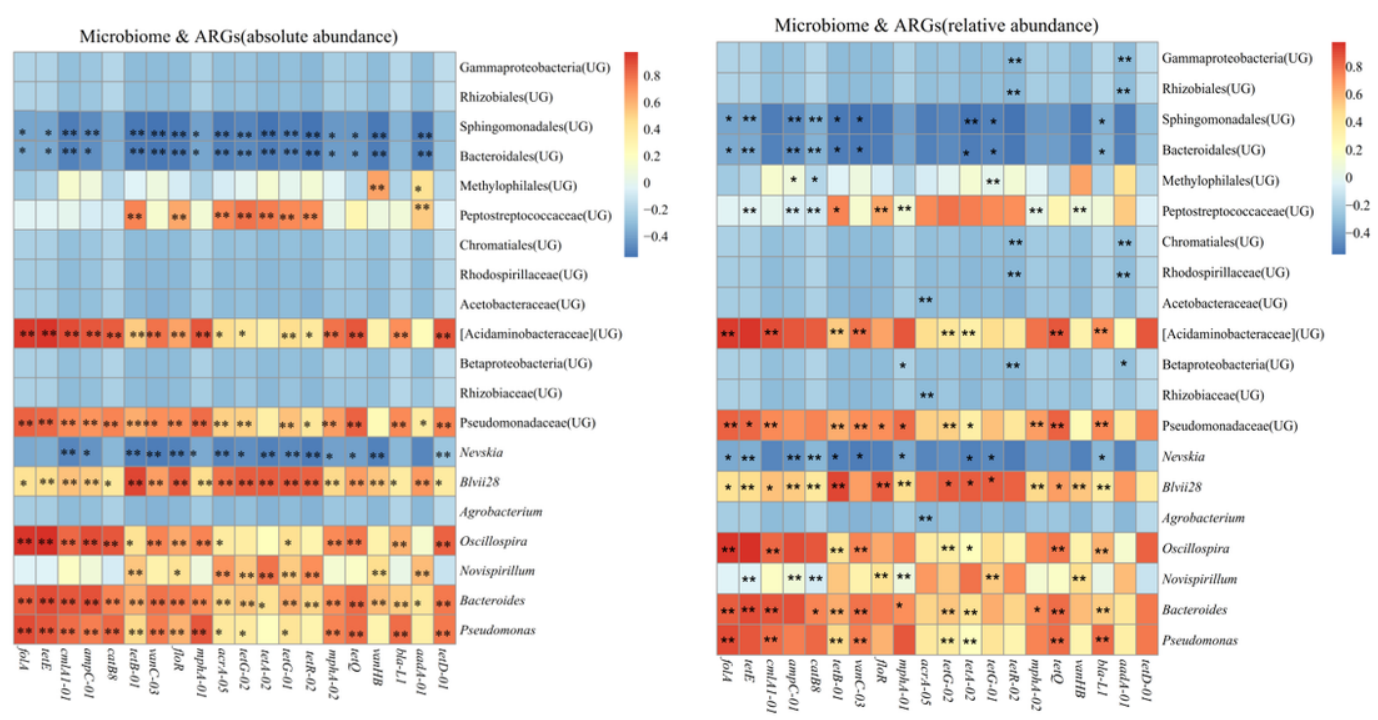

Figure 6

Heatmap showing Spearman's rank correlations between environmental factors and significant ARGs (a-b), and dominant genera with mean relative abundance more than $1 \%$ and significant ARGs (c-d). Strong correlations are indicated by dark color, whereas weak correlations are indicated by light color. ** $\mathrm{P}<0.01$ and $* \mathrm{P}<0.05$. 
$\mathbf{a}$

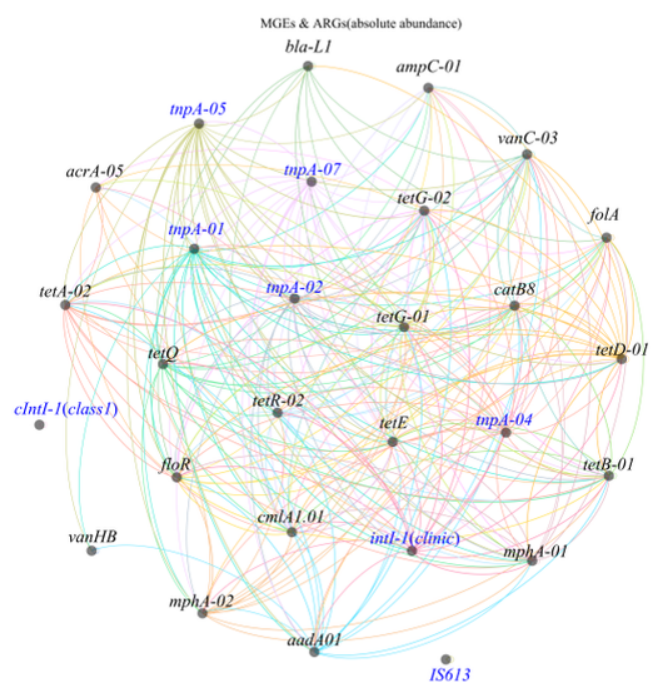

b

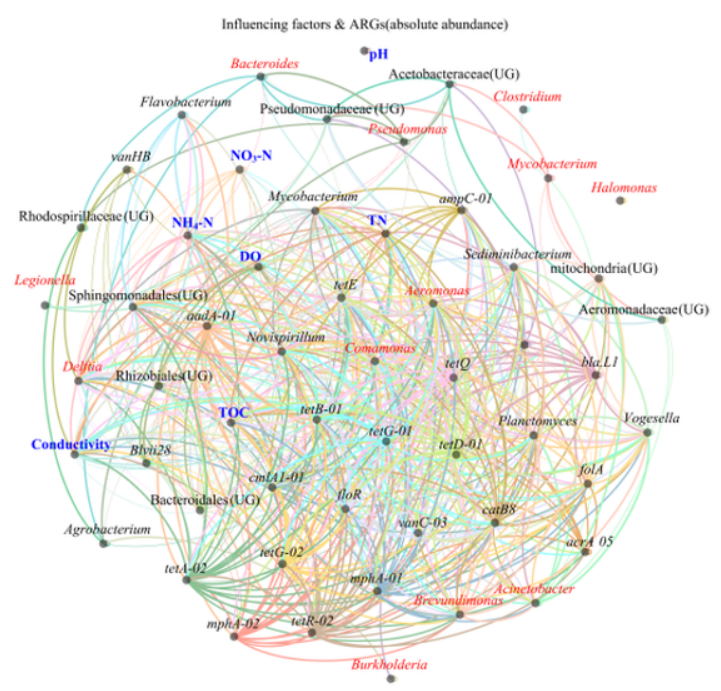

\section{Figure 7}

Network revealing the co-occurrence patterns between the abusolute abundance of MGEs and ARGs (a), and among environmental factors, opportunistic pathogens and ARGs (b). Blue font represents different MGEs (a) and physicochemical factors (b), red font represents opportunistic pathogens, and black font represents ARGs and the main bacterial genera. The edges correspond to significant correlations between nodes $(r>0.6$ or $r<-0.6)$ at $P<0.05$ level.

\section{Supplementary Files}

This is a list of supplementary files associated with this preprint. Click to download.

- SWHsupplement.docx 